Copyright by the Acoustical Society of America. Shaarawi, A. M., Besieris, I. M., Attiya, A. M., \& El-Diwany, E. (2000). Acoustic X-wave reflection and transmission at a planar interface: Spectral analysis. Journal of the Acoustical Society of America, 107(1), 70-86. doi: 10.1121/1.428345

\title{
Acoustic X-wave reflection and transmission at a planar interface: Spectral analysis
}

\author{
Amr M. Shaarawi \\ Department of Engineering Physics and Mathematics, Faculty of Engineering, Cairo University, \\ Giza 12211, Egypt
}

loannis M. Besieris

The Bradley Department of Electrical and Computer Engineering, Virginia Polytechnic Institute and State University, Blacksburg, Virginia 24061

Ahmed M. Attiya and Essam El-Diwany

Department of Microwave Engineering, Electronic Research Institute, Dokki, Giza, 12211, Egypt

(Received 5 March 1999; revised 31 August 1999; accepted 2 September 1999)

\begin{abstract}
The spectral structure of a three-dimensional X-wave pulse incident on a planar surface of discontinuity is examined. Introducing a novel superposition of azimuthally dependent pulsed plane waves, it is shown for oblique incidence that the reflected pulse has a localized wave structure. On the other hand, the transmitted field maintains its localization up to a certain distance from the interface, beyond which it starts disintegrating. An estimate of the localization range of the transmitted pulse is established; also, the parameters affecting the localization range are identified. The reflected and transmitted fields are deduced for X-waves incident from either a slower medium or a faster one. For the former case the evanescent fields in the second medium are calculated and their explicit time dependence is deduced for a normally incident $\mathrm{X}$-wave. Furthermore, at near-critical incidence the transmitted pulse exhibits significant pulse compression and focusing. (C) 2000 Acoustical Society of America. [S0001-4966(99)05412-0]
\end{abstract}

PACS numbers: 43.20.Bi, 43.20.Px [ANN]

\section{INTRODUCTION}

Studies of the generation of acoustical and optical localized waves $(\mathrm{LW})^{1-7}$ and their use in high-resolution imaging and target identification ${ }^{8-10}$ have advanced considerably in the past few years. Because of their large focusing depths and their wideband spectra, LWs have a potential in detecting objects buried at different depths and identifying wide ranges of the parameters characterizing a detected target. ${ }^{8} \mathrm{~A}$ large number of different LW solutions have appeared in literature; they can be divided into two distinct categories. One category consists of solutions based on Brittingham's focus wave modes (FWM) ${ }^{11-14}$ while the other is based on the X-waves deduced by $\mathrm{Lu}$ and Greenleaf. ${ }^{15-18} \mathrm{~A}$ common attribute of these two categories of LWs is the spectral coupling between their spatial and temporal frequency components. This specific property characterizes LWs and differentiates them from other broadband pulses. In particular, such coupling causes the spectral depletion of radiated LWs to be different from that of other types of broadband fields. ${ }^{19}$ It has been shown recently that the focus wave modes and the $\mathrm{X}$-waves have the same spectral structure except that their spatio-temporal couplings are different. ${ }^{20,21}$ Whereas the $\mathrm{X}$-waves have a coupling for which the temporal frequency is proportional to the spatial frequency, the field of the FWMs is characterized by a temporal frequency that is proportional to the square of the spatial frequency.

In many applications, one expects acoustic localized waves to be transmitted from one medium to another. It is, therefore, important to study the details of a canonical problem involving the reflection and transmission of a localized wave obliquely incident on a planar interface separating two acoustically different materials. A similar problem has been investigated previously in two different papers. ${ }^{22,23}$ In both cases, the authors used a two-dimensional variation of the Brittingham's FWM electromagnetic solution. In the first paper, Hillion has argued that FWMs are transmitted across a discontinuity surface only if they are normally incident on that surface, while for oblique incidence only reflection takes place. ${ }^{22}$ Furthermore, he has demonstrated that for normal incidence the transmitted, reflected and incident fields have the same structure. Hillion's results have been criticized by Donnelly and Power. ${ }^{23}$ They have used a geometrical approach to prove that the transmitted waveform of the twodimensional FWM does not have a LW structure. In their approach, LW solutions are represented in the form of a space-time Fourier transform whose domain is constrained to a specific geometrical support. This is essentially a geometrical interpretation of the spectral coupling between the spatial and temporal frequency components. ${ }^{7,19-21}$ Donnelly and Power have argued that for oblique incidence the reflected waveform has an LW structure, while the transmitted waveform does not. The aim of this paper is to clarify this point and to quantify the process of disintegration of the transmitted LW field.

In this work, we will investigate the case of reflection and transmission of the three-dimensional acoustic X-waves. There are several advantages associated with this choice, in contrast to working with vector fields or carrying out the analysis in two dimensions. In a three-dimensional framework, the pulses do not exhibit the extended tails appearing 
in two-dimensional pulsed fields. ${ }^{24,25}$ Trailing fields associated with 2-D pulses can obscure some of the wave phenomena associated with the reflection and transmission of transients. ${ }^{25}$ In addition, the X-wave has a simpler spectral structure than the FWM solution and contains no backward traveling (acausal) components. Such acausal components can give rise to serious conceptual difficulties when dealing with an LW pulse incident on a planar interface. Furthermore, the study of transmission and reflection of a scalar LW pulse is free from the complexity associated with the polarization properties of an analogous vector field. This makes the mathematics more manageable and allows us to clearly identify the factors affecting the disintegration of the transmitted field. Finally, the peaks of a scalar X-wave lie on the axis of propagation, while those of the corresponding electromagnetic field components are located off-axis. Thus the decay of the peak of a scalar acoustic X-wave pulse is more tractable than that of an electromagnetic X-wave pulse.

In this paper, we elaborate on the spectral structure of LWs incident on an interface separating two different media. The analysis used in this paper follows basically that of Donnelly and Power; however, it is applied to the threedimensional scalar X-wave solution instead of the twodimensional vector FWM field. We show that for oblique incidence, the reflected $\mathrm{X}$-wave has an LW structure. We also provide an explanation for the cause of disintegration of the transmitted field. This explanation is based on a spectral analysis that leads to a representation involving an integration of an angular distribution of azimuthally dependent elementary pulses. For the incident and reflected X-waves, all the azimuthally dependent pulses travel at the same speed and add up coherently at any observation point synthesizing a diffraction-free LW pulse. In contradistinction, the transmitted azimuthally dependent pulses travel at different speeds. The integration of such dispersed pulses results in a wave field having an axial width that increases with distance from the interface. This causes the central part of the transmitted pulse to become longer, and the amplitude of its peak to decrease with distance. The analysis used in this paper reveals that the transmitted field stays localized for a certain range, beyond which it starts to disintegrate. The disintegration of the transmitted wave field starts at a depth that depends on several parameters characterizing the incident pulse. The main goal of this work is to demonstrate how to estimate this localization depth and to identify the parameters that determine such a range.

The plan of this work is as follows: A Fourier spectral representation of the incident pulse is introduced in Sec. I. This is done for a 3-D X-wave incident normally on the planar interface separating two media. In the same section, we show that an obliquely incident $\mathrm{X}$-wave can be derived by rotating the normally incident pulse. Expressions for the Fourier composition of both the reflected and transmitted pulses are deduced in Sec. II. The Fourier integrals associated with the incident, reflected and transmitted fields are transformed into azimuthal angular superpositions over elementary pulsed solutions of the scalar wave equation. These azimuthal angular representations are then used in Sec. III to explain the localization of the reflected LW field and the

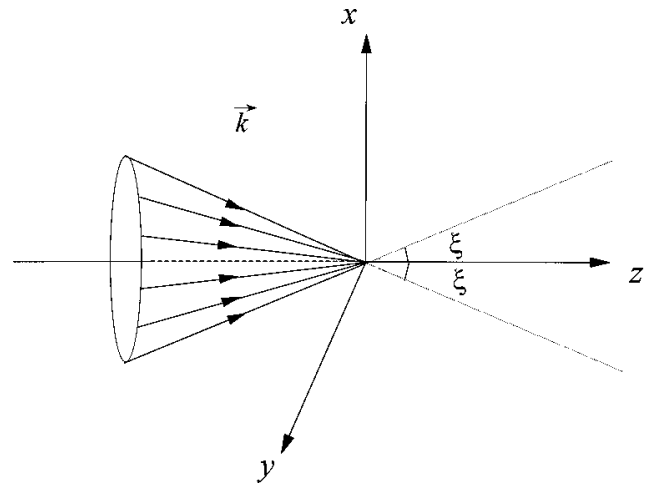

FIG. 1. Wave vectors of the normally incident $\mathrm{X}$-wave lying on a conic surface having an apex angle $\xi$.

reason for the disintegration of the transmitted field. The dispersion of the transmitted pulse is quantified by deducing an expression for a dispersion-free distance in the second medium. In Sec. IV, we provide a detailed numerical study of the behavior of the reflected and transmitted fields, including situations involving total reflection and the generation of evanescent fields. This investigation shows that the dispersion-free distance deduced in Sec. III is fairly accurate.

\section{THE SPECTRAL COMPOSITION OF THE INCIDENT X-WAVE}

In this section, we study the spectral composition of an acoustic X-wave pulse incident on an interface separating two different media. The spectral wave components will be used in later sections to study the behavior of the reflected and transmitted fields. The X-wave pressure field is a solution of the 3-D scalar wave equation

$$
\left(\nabla^{2}-c^{-2} \partial_{t}^{2}\right) p(\mathbf{r}, t)=0,
$$

where $c=\sqrt{B / \rho_{0}}$ is the wave velocity in the medium, $B$ being the bulk modulus of the medium and $\rho_{0}$ its density. We will restrict our analysis to compressive waves only; i.e., shear components will be neglected throughout the paper. This treatment models a large number of situations, e.g., an air-fluid or a gas-gas interface.

$\mathrm{X}$-waves are wide-band pulses synthesized of plane waves components characterized by wave vectors lying on a conical surface defined by the apex angle $\theta_{k}=\xi$ in momentum space. ${ }^{26,27}$ The superposition of such plane waves produces an interference pattern moving with the supersonic speed $\nu=c / \cos \xi$, although each individual plane wave propagates at the phase speed of the medium $c$ along the cone surface. ${ }^{26,27}$ An $\mathrm{X}$-wave traveling in the positive $z$-direction has wave vectors forming the conical surface shown in Fig. 1. This represents the situation of normal incidence on an interface of discontinuity situated at $z=0$. For oblique incidence, the cone shown in Fig. 1 must be rotated by an angle equal to the angle of incidence of the $\mathrm{X}$-wave pulse. Sections of the tilted incident, reflected and transmitted cones taken at $x=0$ are shown in Fig. 2. We will start with a normally incident $\mathrm{X}$-wave propagating in the positive $z$-direction. Then, we will rotate this solution to obtain an obliquely incident $\mathrm{X}$-wave. 


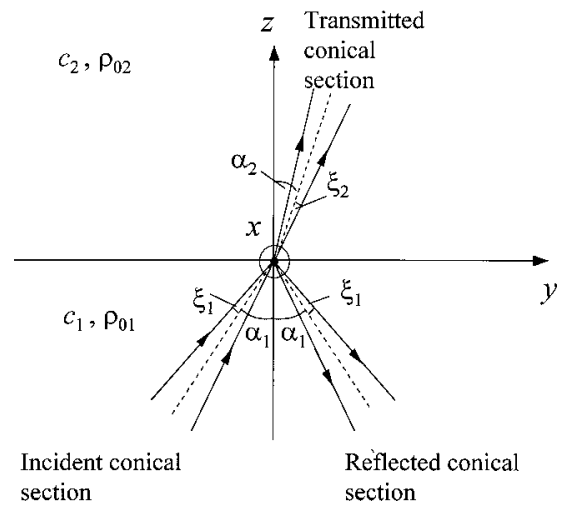

FIG. 2. Longitudinal sections of the spectral cones of the incident, reflected and transmitted fields. The surface of discontinuity between the two media is situated at the $z=0$ plane.

We commence our work by introducing the incident pressure field, which is represented by the fourfold Fourier transform of the zeroth order X-wave solution. Specifically,

$$
\begin{aligned}
p_{\text {inc }}(\mathbf{r}, t)= & \int_{R^{3}} d^{3} \mathbf{k} \int_{0}^{\infty} d\left(\omega / c_{1}\right) e^{-i \mathbf{k} \cdot \mathbf{r}} e^{+i \omega t} A_{\text {inc }}(\mathbf{k}, \omega) \\
& \times \delta\left(k_{z}-\left(\omega / c_{1}\right) \cos \xi_{1}\right) \\
& \times \delta\left(k_{x}^{2}+k_{y}^{2}-\left(\omega / c_{1}\right)^{2} \sin ^{2} \xi_{1}\right) .
\end{aligned}
$$

Here, $A_{\text {inc }}(\mathbf{k}, \omega)=A_{0} e^{-\left(\omega / c_{1}\right) a}, a$ is a real positive parameter, $A_{0}$ is a constant spectral amplitude, the wave velocity in the first medium is $c_{1}$ and $\delta(\cdot)$ denotes the Dirac delta function. The integration over $k_{x}$ and $k_{z}$ yields

$$
\begin{aligned}
p_{\text {inc }}(\mathbf{r}, t)= & \int_{0}^{\infty} d\left(\omega / c_{1}\right) \int_{-\left(\omega / c_{1}\right) \sin \xi_{1}}^{+\left(\omega / c_{1}\right) \sin \xi_{1}} d k_{y} \\
& \times \frac{A_{0} e^{-\left(\omega / c_{1}\right) a}}{\sqrt{\left(\omega / c_{1}\right)^{2} \sin ^{2} \xi_{1}-k_{y}^{2}}}
\end{aligned}
$$

$$
\begin{aligned}
& \times \cos \left(x \sqrt{\left(\omega / c_{1}\right)^{2} \sin ^{2} \xi_{1}-k_{y}^{2}}\right) \\
& \times e^{-i k_{y} y} e^{-i\left(\omega / c_{1}\right) \cos \xi_{1} z} e^{+i \omega t} .
\end{aligned}
$$

The range of integration over $k_{y}$ reflects the fact that $\mathrm{X}$-waves have no evanescent fields. The integration over $k_{y}$ in Eq. (3) yields (cf. 3.876.7 in Ref. 28)

$$
\begin{aligned}
p_{\text {inc }}(\mathbf{r}, t)= & \pi \int_{0}^{\infty} d\left(\omega / c_{1}\right) J_{0}\left(\left(\omega / c_{1}\right) \rho \sin \xi_{1}\right) \\
& \times A_{0} e^{-\left(\omega / c_{1}\right) a} e^{-i\left(\omega / c_{1}\right)\left(z \cos \xi_{1}-c_{1} t\right)} .
\end{aligned}
$$

Finally, the integration over $\left(\omega / c_{1}\right)$ gives rise to the result

$$
p_{\text {inc }}(\mathbf{r}, t)=\frac{\pi A_{0}}{\left[\rho^{2} \sin ^{2} \xi_{1}+\left(a+i\left(z \cos \xi_{1}-c_{1} t\right)\right)^{2}\right]^{1 / 2}} .
$$

This is a localized pulse traveling in the positive $z$-direction with a peaked center propagating at the supersonic speed $c_{1} / \cos \xi_{1}$.

For obliquely incident $\mathrm{X}$-waves, we need to rotate either the coordinates $(x, y, z)$ or the wave vector components $\left(k_{x}, k_{y}, k_{z}\right)$. A clockwise rotation by an angle $\alpha_{1}$ around the $x$-axis is defined by the operation $\mathbf{r}^{\prime}=M \mathbf{r}$, where

$$
M=\left[\begin{array}{ccc}
1 & 0 & 0 \\
0 & \cos \alpha_{1} & -\sin \alpha_{1} \\
0 & \sin \alpha_{1} & \cos \alpha_{1}
\end{array}\right]
$$

This operation transforms a normally incident $\mathrm{X}$-wave to the following obliquely incident one:

$$
p_{\text {inc }}(\mathbf{r}, t)=\frac{\pi A_{0}}{\left[x^{2} \sin ^{2} \xi_{1}+\left(y \cos \alpha_{1}-z \sin \alpha_{1}\right)^{2} \sin ^{2} \xi_{1}+\left(a+i\left(\left(y \sin \alpha_{1}+z \cos \alpha_{1}\right) \cos \xi_{1}-c_{1} t\right)\right)^{2}\right]^{1 / 2}} .
$$

Alternatively, we can rotate the components of the wave vector $\mathbf{k}$ using the procedure described by Donnelly and Power. ${ }^{23}$ Specifically, the Fourier superposition of a rotated $\mathrm{X}$-wave

$$
p_{\text {inc }}(M \mathbf{r}, t)=\int_{R^{3}} d^{3} \mathbf{k} \int_{R} d \omega e^{-i \mathbf{k} \cdot M \mathbf{r}} e^{i \omega t} \widetilde{p}_{\text {inc }}(\mathbf{k}, \omega)
$$

is equivalent to having

$$
p_{\text {inc }}(M \mathbf{r}, t)=\int_{R^{3}} d^{3} \mathbf{k}^{\prime} \int_{R} d \omega e^{-i \mathbf{k}^{\prime} \cdot \mathbf{r}} e^{i \omega t} \widetilde{p}_{\text {inc }}\left(\mathbf{k}^{\prime}, \omega\right),
$$

where $\mathbf{k}^{\prime}=M^{T} \mathbf{k}$ and $\mathbf{k}=M \mathbf{k}^{\prime}$. Substituting these relations into the Fourier superposition Eq. (2) gives the following expression:

$$
\begin{aligned}
p_{\text {inc }}(\mathbf{r}, t)= & \int_{0}^{\infty} d\left(\omega / c_{1}\right) \int_{R^{3}} d^{3} \mathbf{k}^{\prime} e^{-i \mathbf{k}^{\prime} \cdot \mathbf{r}} e^{i \omega t} \\
& \times A_{0} e^{-\left(\omega / c_{1}\right) a} \delta\left(k_{y}^{\prime} \sin \alpha_{1}+k_{z}^{\prime} \cos \alpha_{1}\right. \\
& \left.-\left(\omega / c_{1}\right) \cos \xi_{1}\right) \delta\left(k_{x}^{\prime 2}\right. \\
& \left.+\left(k_{y}^{\prime} \cos \alpha_{1}-k_{z}^{\prime} \sin \alpha_{1}\right)^{2}-\left(\omega / c_{1}\right)^{2} \sin ^{2} \xi_{1}\right) .
\end{aligned}
$$

Integrating over $k_{x}^{\prime}$ and $k_{z}^{\prime}$, the above expression reduces to 


$$
\begin{aligned}
p_{\text {inc }}(\mathbf{r}, t)= & \int_{0}^{\infty} d\left(\omega / c_{1}\right) \int_{\Delta_{-}}^{\Delta_{+}} d k_{y}^{\prime} \\
& \times \frac{A_{0} e^{-\left(\omega / c_{1}\right) a}}{\cos \alpha_{1} \sqrt{\left(\omega / c_{1}\right)^{2} \sin ^{2} \xi_{1}-\eta_{1}^{2}}} \\
& \times \cos \left(x \sqrt{\left(\omega / c_{1}\right)^{2} \sin ^{2} \xi_{1}-\eta_{1}^{2}}\right) e^{-i k_{y}^{\prime} y} \\
& \times e^{i z\left(k_{y}^{\prime} \sin \alpha_{1} / \cos \alpha_{1}-\left(\omega / c_{1}\right) \cos \xi_{1} / \cos \alpha_{1}\right)} e^{i \omega t} .
\end{aligned}
$$

Here, we have used the simultaneous roots of the two Dirac delta functions; specifically,

$$
k_{z}^{\prime}=\left(\left(\omega / c_{1}\right) \cos \xi_{1}-k_{y}^{\prime} \sin \alpha_{1}\right) / \cos \alpha_{1}
$$

and

$$
k_{x}^{\prime 2}=\left(\omega / c_{1}\right)^{2} \sin ^{2} \xi_{1}-\eta_{1}^{2},
$$

where

$$
\begin{aligned}
\eta_{1} & =k_{y}^{\prime} \cos \alpha_{1}-k_{z}^{\prime} \sin \alpha_{1} \\
& =\left(k_{y}^{\prime} / \cos \alpha_{1}\right)-\left(\omega / c_{1}\right) \sin \alpha_{1} \cos \xi_{1} / \cos \alpha_{1} .
\end{aligned}
$$

The limits of the integration over $k_{y}^{\prime}$ can be determined by noting that $\left|k_{y}\right|<\left(\omega / c_{1}\right) \sin \xi_{1}$. Hence, $k_{y}^{\prime}=k_{y} \cos \alpha_{1}$ $+k_{z} \sin \alpha_{1}$ is limited so that $-\left(\omega / c_{1}\right) \sin \xi_{1} \cos \alpha_{1}$ $+\left(\omega / c_{1}\right) \cos \xi_{1} \sin \alpha_{1}<k_{y}^{\prime}<\left(\omega / c_{1}\right) \sin \xi_{1} \cos \alpha_{1}+\left(\omega / c_{1}\right) \cos \xi_{1}$ $\times \sin \alpha_{1}$. Consequently, the two limits of the integration over $k_{y}^{\prime}$ in Eq. (9) are given by $\Delta_{ \pm}=\left(\omega / c_{1}\right) \sin \left(\alpha_{1} \pm \xi_{1}\right)$.

Introducing the change of variables $k_{y}^{\prime} \rightarrow \eta_{1}$ and rearranging terms, Eq. (9) can be rewritten as

$$
\begin{aligned}
p_{\text {inc }}(\mathbf{r}, t)= & \int_{0}^{\infty} d\left(\omega / c_{1}\right) \int_{-\left(\omega / c_{1}\right) \sin \xi_{1}}^{+\left(\omega / c_{1}\right) \sin \xi_{1}} d \eta_{1} \\
& \times \frac{A_{0} e^{-\left(\omega / c_{1}\right) a}}{\sqrt{\left(\omega / c_{1}\right)^{2} \sin ^{2} \xi_{1}-\eta_{1}^{2}}} \\
& \times \cos \left(x \sqrt{\left(\omega / c_{1}\right)^{2} \sin ^{2} \xi_{1}-\eta_{1}^{2}}\right) \\
& \times e^{i \omega t} e^{-i \eta_{1}\left(y \cos \alpha_{1}-z \sin \alpha_{1}\right)} \\
& \times e^{-i\left(\omega / c_{1}\right) \cos \xi_{1}\left(y \sin \alpha_{1}+z \cos \alpha_{1}\right)} .
\end{aligned}
$$

Comparing Eqs. (10) and (3), we find that the above integration yields the same obliquely incident $\mathrm{X}$-wave as that given in Eq. (7). We have, thus, demonstrated that we can change the direction of propagation of the X-wave by either rotating the position coordinates or by rotating the components of the wave vector $\mathbf{k}$. In later sections, the real part of $p_{\text {inc }}(\mathbf{r}, t)$ will be used to represent the differential pressure of the incident acoustical field. The same criterion is applied in case of complex amplitudes representing reflected and transmitted pressure fields. The real parts of the pressure amplitudes have distinct peaks that are easy to trace. For such reason, we have preferred to use them in our study rather than the imaginary parts.

\section{SPECTRAL REPRESENTATION OF THE REFLECTED AND TRANSMITTED X-WAVES}

Consider the case of an acoustical X-wave obliquely incident on a surface of discontinuity separating two different media. Assume that the interface separating the two media is situated at $z=0$. Suppose that the first medium has a wave velocity $c_{1}$ and a density $\rho_{01}$, and that the second medium has a wave velocity $c_{2}$ and a density $\rho_{02}$. The axis of propagation of the $\mathrm{X}$-wave incident from the negative $z$ hemisphere makes an angle $\alpha_{1}$ with the normal to the interface, as shown in Fig. 2. If the incident X-wave field has wave vector k components $k_{x \text { inc }}, k_{y \text { inc }}$ and $k_{z \text { inc }}$, then the components of the reflected and transmitted wave vectors become

$$
\begin{aligned}
& k_{x \mathrm{ref}}=k_{x \mathrm{inc}}, \quad k_{y \mathrm{ref}}=k_{y \mathrm{inc}} \text { and } k_{z \mathrm{ref}}=-k_{z \mathrm{inc}} ; \\
& k_{x \operatorname{tran}}=k_{x \mathrm{inc}}, \quad k_{y \operatorname{tran}}=k_{y \mathrm{inc}} \text { and } \\
& k_{z \operatorname{tran}}=\sqrt{\left(\omega / c_{2}\right)^{2}-k_{z \mathrm{inc}}^{2}-k_{y \mathrm{inc}}^{2}} .
\end{aligned}
$$

Suppose that the pressure wave associated with a normally incident field is represented by the expression given in Eq. (2). The corresponding reflected and transmitted fields are given by

$$
\begin{aligned}
p_{\text {ref }}(\mathbf{r}, t)= & \int_{R^{3}} d^{3} \mathbf{k} \int_{0}^{\infty} d\left(\omega / c_{1}\right) e^{-i \mathbf{k} \cdot \mathbf{r}} e^{+i \omega t} A_{\text {ref }}(\mathbf{k}, \omega) \\
& \times \delta\left(k_{z}+\left(\omega / c_{1}\right) \cos \xi_{1}\right) \\
& \times \delta\left(k_{x}^{2}+k_{y}^{2}-\left(\omega / c_{1}\right)^{2} \sin ^{2} \xi_{1}\right)
\end{aligned}
$$

and

$$
\begin{aligned}
p_{\text {tran }}(\mathbf{r}, t)= & \int_{R^{3}} d^{3} \mathbf{k} \int_{0}^{\infty} d\left(\omega / c_{2}\right) e^{-i \mathbf{k} \cdot \mathbf{r}} e^{+i \omega t} \\
& \times A_{\operatorname{tran}}(\mathbf{k}, \omega) \delta\left(k_{z}-\left(\omega / c_{2}\right) \cos \xi_{2}\right) \\
& \times \delta\left(k_{x}^{2}+k_{y}^{2}-\left(\omega / c_{2}\right)^{2} \sin ^{2} \xi_{2}\right) .
\end{aligned}
$$

The apex angle of the spectral cones of the incident and transmitted X-waves are denoted by $\xi_{1}$ and $\xi_{2}$, respectively. In Eq. (14), the conditions $k_{x \operatorname{tran}}=k_{x \text { inc }}$ and $k_{y \operatorname{tran}}=k_{y \text { inc }}$ are satisfied because from Snell's law $\left(\omega / c_{2}\right) \sin \xi_{2}$ $=\left(\omega / c_{1}\right) \sin \xi_{1}$. The condition $k_{z}=\left(\omega / c_{2}\right) \cos \xi_{2}$ imposed by the other Dirac delta function is another way of writing the relation $k_{z \operatorname{tran}}=\sqrt{\left(\omega / c_{2}\right)^{2}-k_{z \text { inc }}^{2}-k_{y \text { inc }}^{2}}$. Notice that for normal incidence, the reflected and transmitted pressure fields have the same spectral structures as those of the incident field. Consequently, the reflected and transmitted fields due to a normally incident $\mathrm{X}$-wave retain their LW structure.

The continuity conditions of the pressure and the normal displacement components at the boundary give the following relations between the spectral weighting functions ${ }^{29,30}$

$$
\begin{aligned}
& A_{\text {ref }}=A_{\text {inc }} \frac{Z_{2} \sec \theta_{2 k}-Z_{1} \sec \theta_{1 k}}{Z_{2} \sec \theta_{2 k}+Z_{1} \sec \theta_{1 k}}, \\
& A_{\text {tran }}=A_{\text {inc }} \frac{2 Z_{2} \sec \theta_{2 k}}{Z_{2} \sec \theta_{2 k}+Z_{1} \sec \theta_{1 k}} .
\end{aligned}
$$

Here, the characteristic wave impedances for the two media are defined as $Z_{1} \equiv \rho_{01} c_{1}$ and $Z_{2} \equiv \rho_{02} c_{2}$. The angles between the $z$-axis and the wave vectors associated with the individual components contributing to the incident and transmitted fields are denoted by $\theta_{1 k}$ and $\theta_{2 k}$, respectively. For normal incidence, these angles equal the apex angles $\xi_{1}$ and $\xi_{2}$. Before we substitute the reflection and transmission co- 
efficients in the Fourier representations of the various fields, we need to express them in terms of the wave vectors, viz.,

$$
A_{\text {ref }}=A_{\text {inc }} \frac{k_{z \text { inc }}-k_{z \operatorname{tran}}\left(\rho_{01} / \rho_{02}\right)}{k_{z \text { inc }}+k_{z \operatorname{tran}}\left(\rho_{01} / \rho_{02}\right)},
$$

$$
A_{\text {tran }}=A_{\text {inc }} \frac{2 k_{z \text { inc }}}{k_{z \text { inc }}+k_{z \operatorname{tran}}\left(\rho_{01} / \rho_{02}\right)} .
$$

Therefore, for an obliquely incident $\mathrm{X}$-wave, at an angle of inclination $\alpha_{1}$, the reflected pressure field acquires the form

$$
\begin{aligned}
p_{\text {ref }}(\mathbf{r}, t)= & \int_{0}^{\infty} d\left(\omega / c_{1}\right) \int_{R^{3}} d^{3} \mathbf{k}^{\prime} e^{-i \mathbf{k}^{\prime} \cdot \mathbf{r}} e^{+i \omega t} A_{0} e^{-\left(\omega / c_{1}\right) a} \frac{k_{z}^{\prime}-\operatorname{sgn}\left(k_{z}^{\prime}\right) \sqrt{\left(\omega / c_{2}\right)^{2}-k_{x}^{\prime 2}-k_{y}^{\prime 2}}\left(\rho_{01} / \rho_{02}\right)}{k_{z}^{\prime}+\operatorname{sgn}\left(k_{z}^{\prime}\right) \sqrt{\left(\omega / c_{2}\right)^{2}-k_{x}^{\prime 2}-k_{y}^{\prime 2}}\left(\rho_{01} / \rho_{02}\right)} \\
& \times \delta\left(-k_{z}^{\prime} \cos \alpha_{1}+k_{y}^{\prime} \sin \alpha_{1}-\left(\omega / c_{1}\right) \cos \xi_{1}\right) \delta\left(k_{x}^{\prime 2}+\left(k_{y}^{\prime} \cos \alpha_{1}+k_{z}^{\prime} \sin \alpha_{1}\right)^{2}-\left(\omega / c_{1}\right)^{2} \sin ^{2} \xi_{1}\right)
\end{aligned}
$$

where we have rotated the $\mathbf{k}$ vector and used the relation between $A_{\text {ref }}\left(\mathbf{k}^{\prime}, \omega\right)$ and $A_{\text {inc }}\left(\mathbf{k}^{\prime}, \omega\right)$ given in Eq. (17). Notice that the transformation $k_{z}^{\prime} \rightarrow-k_{z}^{\prime}$ in the arguments of the delta functions guarantees that the wave vectors are reflected in the correct direction. The delta functions in Eq. (19) imply that

$$
k_{z}^{\prime}=\eta_{1} \sin \alpha_{1}-\left(\omega / c_{1}\right) \cos \xi_{1} \cos \alpha_{1},
$$

and the argument of the square root contributing to the reflection coefficient becomes

$$
\left(\omega / c_{2}\right)^{2}-k_{x}^{\prime 2}-k_{y}^{\prime 2}=\left(\omega / c_{2}\right)^{2}-\left(\omega / c_{1}\right)^{2}+\Lambda_{1}^{2}(\omega),
$$

where $\Lambda_{1}(\omega)=\eta_{1} \sin \alpha_{1}-\left(\omega / c_{1}\right) \cos \alpha_{1} \cos \xi_{1}$. Notice that for $\alpha_{1}$ and $\xi_{1}$ values obeying the condition $\alpha_{1}+\xi_{1}<\pi / 2$, the quantity $\Lambda_{1}(\omega)$ is negative. This means that $\operatorname{sgn}\left(k_{z}^{\prime}\right)$ is negative as expected for the reflected field. Carrying out the integration over $k_{x}^{\prime}$ and $k_{z}^{\prime}$ in Eq. (19) and changing the integration variable $k_{y}^{\prime} \rightarrow \eta_{1}$, we obtain

$$
\begin{aligned}
p_{\text {ref }}(\mathbf{r}, t)= & \int_{0}^{\infty} d\left(\omega / c_{1}\right) \int_{-\omega / c \sin \xi}^{\omega / c_{1} \sin \xi} d \eta_{1} \frac{A_{0} e^{-\left(\omega / c_{1}\right) a}}{\sqrt{\left(\omega / c_{1}\right)^{2} \sin ^{2} \xi_{1}-\eta_{1}^{2}}} \cos \left(x \sqrt{\left(\omega / c_{1}\right)^{2} \sin ^{2} \xi_{1}-\eta_{1}^{2}}\right) \\
& \times e^{-i \eta_{1}\left(y \cos \alpha_{1}+z \sin \alpha_{1}\right)} e^{-i\left(\omega / c_{1}\right) \cos \xi_{1}\left(y \sin \alpha_{1}-z \cos \alpha_{1}\right)} e^{i \omega t} R\left(\eta_{1}, \omega\right),
\end{aligned}
$$

where the reflection coefficient may be rewritten as

$$
R\left(\eta_{1}, \omega\right)=\frac{\Lambda_{1}(\omega)+\sqrt{\left(\omega / c_{2}\right)^{2}-\left(\omega / c_{1}\right)^{2} \sin ^{2} \xi_{1}+\eta_{1}^{2}-\Xi_{1}^{2}(\omega)}\left(\rho_{01} / \rho_{02}\right)}{\Lambda_{1}(\omega)-\sqrt{\left(\omega / c_{2}\right)^{2}-\left(\omega / c_{1}\right)^{2} \sin ^{2} \xi_{1}+\eta_{1}^{2}-\Xi_{1}^{2}(\omega)}\left(\rho_{01} / \rho_{02}\right)},
$$

with $\Xi_{1}(\omega)=\eta_{1} \cos \alpha_{1}+\left(\omega / c_{1}\right) \sin \alpha_{1} \cos \xi_{1}$. The integration in Eq. (20a) cannot be evaluated explicitly in order to obtain a closed-form expression for the reflected pressure field and will be considered further in the next section.

The same procedure described above can be repeated in order to calculate the transmitted field. We have to be sure, however, that the continuity condition Eq. (12) is valid for the incident and transmitted plane wave components. Along this vein, consider Snell's law

$$
\left(1 / c_{1}\right) \sin \theta_{1 k}=\left(1 / c_{2}\right) \sin \theta_{2 k},
$$

where $\theta_{1 k}$ and $\theta_{2 k}$ are the angles of the wave vectors associated with the incident and transmitted plane wave components, respectively. In particular,

$$
\cos \theta_{1 k}=\left(k_{z 1}^{\prime} /\left(\omega / c_{1}\right)\right)=\cos \xi_{1} \cos \alpha_{1}-\eta_{1} \sin \alpha_{1} /\left(\omega / c_{1}\right) .
$$

Substituting Eq. (22) into Eq. (23), Snell's law may be rewritten as follows:

$$
\cos ^{2} \theta_{2 k}=1-\left(c_{2} / c_{1}\right)^{2}\left(1-\cos ^{2} \theta_{1 k}\right)=1-\left(c_{2} / c_{1}\right)^{2}\left(1-\left(\cos \xi_{1} \cos \alpha_{1}-\eta_{1} \sin \alpha_{1} /\left(\omega / c_{1}\right)\right)^{2}\right) .
$$

Hence, the wave number $k_{z 2}^{\prime}$ of the transmitted field is expressed explicitly as

$$
k_{z 2}^{\prime}=\left(\omega / c_{2}\right) \cos \theta_{2 k}=\sqrt{\left(\omega / c_{2}\right)^{2}-\left(\omega / c_{1}\right)^{2}+\left(\left(\omega / c_{1}\right) \cos \xi_{1} \cos \alpha_{1}-\eta_{1} \sin \alpha_{1}\right)^{2}} .
$$

The continuity of the tangential $\mathbf{k}$ components $\left(k_{x \operatorname{tran}}=k_{x \text { inc }}\right)$ yields

$$
k_{x 2}^{\prime 2}=k_{x 1}^{\prime 2}=\left(\omega / c_{1}\right)^{2} \sin ^{2} \xi_{1}-\eta_{1}^{2} .
$$

The Fourier superposition of the transmitted field can thus be reduced to an expression analogous to Eq. (20); specifically, 


$$
\begin{aligned}
p_{\text {trans }}(\mathbf{r}, t)= & \int_{0}^{\infty} d\left(\omega / c_{1}\right) \int_{-\omega / c_{1} \sin \xi}^{\omega / c_{1} \sin \xi} d \eta_{1} \frac{A_{0} e^{-\left(\omega / c_{1}\right) a}}{\sqrt{\left(\omega / c_{1}\right)^{2} \sin ^{2} \xi_{1}-\eta_{1}^{2}}} \cos \left(x \sqrt{\left(\omega / c_{1}\right)^{2} \sin ^{2} \xi_{1}-\eta_{1}^{2}}\right) e^{i \omega t} \\
& \times e^{-i y\left(\eta_{1} \cos \alpha_{1}+\left(\omega / c_{1}\right) \cos \xi_{1} \sin \alpha_{1}\right)} e^{-i z \sqrt{\left(\omega / c_{2}\right)^{2}-\left(\omega / c_{1}\right)^{2}+\left(\left(\omega / c_{1}\right) \cos \xi_{1} \cos \alpha_{1}-\eta_{1} \sin \alpha_{1}\right)^{2}}} T\left(\eta_{1}, \omega\right),
\end{aligned}
$$

where

$$
\begin{aligned}
& T\left(\eta_{1}, \omega\right) \\
& =\frac{2 \Lambda_{1}(\omega)}{\Lambda_{1}(\omega)+\left(\rho_{01} / \rho_{02}\right) \sqrt{\left(\omega / c_{2}\right)^{2}-\left(\omega / c_{1}\right)^{2}+\Lambda_{1}^{2}(\omega)}} .
\end{aligned}
$$

One should point out that the coefficients of $y$ and $z$ in the arguments of the exponential functions cannot be mixed together to form a rotated coordinate. This should be compared to the reflected field where the coefficients of the $y$ and $z$ variables are combined into rotated coordinates that specify the trajectory of the reflected pulse. Similarly to the incident $\mathrm{X}$-wave, wave vectors of the spectral plane wave components of the reflected pulse lie on a single conic surface. In contradistinction, the wave vectors defining the transmitted field components do not form a conic surface. This point has been emphasized in Ref. 31 and is elaborated further in the next section where a new pulsed wave representation is used to illustrate the process of the dispersion of the transmitted field. One should note, however, that for the special case of normal incidence $\alpha_{1}=0$, we have $k_{z 1}=\left(\omega / c_{1}\right) \cos \xi_{1}$ and $k_{z 2}=\left(\omega / c_{2}\right) \cos \xi_{2}$. Consequently, the transmission coefficient given in Eq. (26b) becomes independent of $\omega$ and the integration in Eq. (26a) can be evaluated explicitly to give a dispersion-free X-wave solution.

\section{THE AZIMUTHALLY DEPENDENT PULSE REPRESENTATION}

In this section, we introduce a representation of the incident, reflected and transmitted LW fields using an azi-

muthal angular superposition over elementary pulsed solutions of the scalar wave equation. This framework is then used to explain the localization of the reflected LW field and the reason for the disintegration of the transmitted field. In the preceding section, the quantity $\eta_{1}$ is essentially a rotated $k_{y}$ component. Consequently, the integrations deduced for the incident, reflected and transmitted wave fields can be rewritten in a simpler form by introducing the azimuthal angular variable defined as $\cos \varphi=-\eta_{1} / \kappa_{1} \sin \xi_{1}$, where $\kappa_{1}$ $=\left(\omega / c_{1}\right)$. Noting that $d\left(\omega / c_{1}\right) d \eta_{1}=\kappa_{1} \sin \varphi \sin \xi_{1} d \varphi d \kappa_{1}$, the incident pressure field given in Eq. (10) acquires the following form:

$$
\begin{aligned}
p_{\text {inc }}(\mathbf{r}, t)= & A_{0} \int_{0}^{\infty} d \kappa_{1} \int_{0}^{\pi} d \varphi e^{-\kappa_{1} a} \cos \left(x \kappa_{1} \sin \xi_{1} \sin \varphi\right) \\
& \times e^{i \kappa_{1} \cos \varphi \sin \xi_{1} y^{\prime}} e^{-i \kappa_{1} \cos \xi_{1} z^{\prime}} e^{i \kappa_{1} c_{1} t} .
\end{aligned}
$$

The rotated coordinates associated with the obliquely incident $\mathrm{X}$-wave are $z^{\prime}=y \sin \alpha_{1}+z \cos \alpha_{1}$ and $y^{\prime}=y \cos \alpha_{1}$ $-z \sin \alpha_{1}$. The integration over $\kappa_{1}$ is a Laplace-type that can be easily evaluated to give an angular superposition over the azimuthally dependent pulse $\Psi_{\varphi}(\mathbf{r}, t)$; specifically,

$$
p_{\text {inc }}(\mathbf{r}, t)=\int_{0}^{\pi} d \varphi \Psi_{\varphi}(\mathbf{r}, t),
$$

where

$$
\begin{aligned}
\Psi_{\varphi}(\mathbf{r}, t)= & \frac{A_{0}}{2}\left\{\frac{1}{\left(a+i\left(z^{\prime} \cos \xi_{1}-c_{1} t\right)-i y^{\prime} \sin \xi_{1} \cos \varphi-i x \sin \xi_{1} \sin \varphi\right)}\right. \\
& \left.+\frac{1}{\left(a+i\left(z^{\prime} \cos \xi_{1}-c_{1} t\right)-i y^{\prime} \sin \xi_{1} \cos \varphi+i x \sin \xi_{1} \sin \varphi\right)}\right\} .
\end{aligned}
$$

The angular superposition given in Eq. (28) is effectively a summation of pulses having shapes that depend on the azimuthal angular variable $\varphi$. The integration over $\varphi$ in Eq. (28a) can be evaluated explicitly to yield the X-wave pressure field given in Eq. (7) and whose surface plot is displayed in Fig. 3; for $\alpha_{1}=25^{\circ}, \xi_{1}=2^{\circ}$ and $a=0.001 \mathrm{~m}$. The time dependence of the field is evaluated along the tilted axis of propagation, viz., the quantity $z^{\prime} \cos \xi_{1}-c_{1} t$. The transverse dependence of the field is chosen as a function of $x^{\prime}$ $=x$ by setting the other transverse variable $y^{\prime}=0$. One should note that for such a choice, $z^{\prime}=z / \cos \alpha_{1}$. The field shown in Fig. 3 is calculated at the distance $z=-1 \mathrm{~m}$ from the interface (for which $z^{\prime}=-1 / \cos \alpha_{1}$ ).

Similarly to Eq. (28), the reflected pressure field given in Eq. (20) can be transformed into the form

$$
\begin{aligned}
p_{\text {ref }}(\mathbf{r}, t)= & A_{0} \int_{0}^{\infty} d \kappa_{1} \int_{0}^{\pi} d \varphi e^{-\kappa_{1} a} \cos \left(x \kappa_{1} \sin \xi_{1} \sin \varphi\right) \\
& \times e^{-i \kappa_{1} \cos \varphi \sin \xi_{1} y^{\prime}} e^{-i \kappa_{1} \cos \xi_{1} z^{\prime}} e^{i \kappa_{1} c_{1} t} R(\varphi),
\end{aligned}
$$

where 


$$
R(\varphi)=\frac{\cos \xi_{1} \cos \alpha_{1}+\sin \xi_{1} \sin \alpha_{1} \cos \varphi-\sqrt{\left(c_{1} / c_{2}\right)^{2}-1+\left(\cos \xi_{1} \cos \alpha_{1}+\sin \xi_{1} \sin \alpha_{1} \cos \varphi\right)^{2}}\left(\rho_{01} / \rho_{02}\right)}{\cos \xi_{1} \cos \alpha_{1}+\sin \xi_{1} \sin \alpha_{1} \cos \varphi+\sqrt{\left(c_{1} / c_{2}\right)^{2}-1+\left(\cos \xi_{1} \cos \alpha_{1}+\sin \xi_{1} \sin \alpha_{1} \cos \varphi\right)^{2}}\left(\rho_{01} / \rho_{02}\right)}
$$

is independent of $\kappa_{1}$. The rotated coordinates associated with the reflected wave field are $z^{\prime}=y \sin \alpha_{1}-z \cos \alpha_{1}$ and $y^{\prime}$ $=-y \cos \alpha_{1}-z \sin \alpha_{1}$. The integration over $\kappa_{1}$ leads to an angular superposition which is a generalization of the expression given in Eq. (28); specifically,

$$
p_{\text {ref }}(\mathbf{r}, t)=\int_{0}^{\pi} d \varphi R(\varphi) \hat{\Psi}_{\varphi}(\mathbf{r}, t),
$$

where

$$
\begin{aligned}
\hat{\Psi}_{\varphi}(\mathbf{r}, t)= & \frac{A_{0}}{2}\left\{\frac{1}{\left(a+i\left(z^{\prime} \cos \zeta_{1}-c_{1} t\right)+i y^{\prime} \sin \xi_{1} \cos \varphi-i x \sin \xi_{1} \sin \varphi\right)}\right. \\
& \left.+\frac{1}{\left(a+i\left(z^{\prime} \cos \zeta_{1}-c_{1} t\right)+i y^{\prime} \sin \xi_{1} \cos \varphi+i x \sin \xi_{1} \sin \varphi\right)}\right\} .
\end{aligned}
$$

The expression given in Eq. (30b) indicates that all the reflected azimuthally dependent pulses $\hat{\Psi}_{\varphi}(\mathbf{r}, t)$ travel at the same speed. At any observation point, the pulses $\hat{\Psi}_{\varphi}(\mathbf{r}, t)$ will arrive at the same time to coherently synthesize the reflected field. As a consequence, the reflected field has an LW structure that does not disperse as the pulse travels away from the reflecting interface. This is consistent with having the wave vectors associated with the various plane wave components meet at the apex of a conical surface as explained in Ref. 31 .

The azimuthal angular representation of the transmitted pressure wave can be deduced from Eq. (26) in a similar manner; it is given by

$$
\begin{aligned}
p_{\text {trans }}(\mathbf{r}, t)= & A_{0} \int_{0}^{\infty} d \kappa_{1} \int_{0}^{\pi} d \varphi e^{-\kappa_{1} a} \cos \left(x \kappa_{1} \sin \xi_{1} \sin \varphi\right) e^{i \kappa_{1} y\left(\sin \xi_{1} \cos \alpha_{1} \cos \varphi-\cos \xi \sin \alpha_{1}\right)} \\
& \times e^{-i \kappa_{1} z \sqrt{\left(c_{1} / c_{2}\right)^{2}-1+\left(\cos \xi_{1} \cos \alpha_{1}+\sin \xi_{1} \sin \alpha_{1} \cos \varphi\right)^{2}}} e^{i \kappa_{1} c_{1} t} T(\varphi),
\end{aligned}
$$

where the transmission coefficient

$$
T(\varphi)=\frac{2\left(\cos \xi_{1} \cos \alpha_{1}+\sin \xi_{1} \sin \alpha_{1} \cos \varphi\right)}{\left(\cos \xi_{1} \cos \alpha_{1}+\sin \xi_{1} \sin \alpha_{1} \cos \varphi\right)+\sqrt{\left(c_{1} / c_{2}\right)^{2}-1+\left(\cos \xi_{1} \cos \alpha_{1}+\sin \xi_{1} \sin \alpha_{1} \cos \varphi\right)^{2}}\left(\rho_{01} / \rho_{02}\right)}
$$

is also independent of $\kappa_{1}$. The integration over $\kappa_{1}$ yields

$$
p_{\text {trans }}(\mathbf{r}, t)=\int_{0}^{\pi} d \varphi T(\varphi) \hat{\Psi}_{\varphi}(\mathbf{r}, t)
$$

where

$\hat{\Psi}_{\varphi}(\mathbf{r}, t)$

$$
\begin{aligned}
= & \frac{A_{0}}{2}\left\{\frac{1}{a+i\left(z \sqrt{\left(c_{1} / c_{2}\right)^{2}-1+\left(\cos \xi_{1} \cos \alpha_{1}+\sin \xi_{1} \sin \alpha_{1} \cos \varphi\right)^{2}}-c_{1} t\right)-i y\left(\sin \xi_{1} \cos \alpha_{1} \cos \varphi-\cos \xi_{1} \sin \alpha_{1}\right)-i x \sin \xi_{1} \sin \varphi}\right. \\
& \left.+\frac{1}{a+i\left(z \sqrt{\left(c_{1} / c_{2}\right)^{2}-1+\left(\cos \xi_{1} \cos \alpha_{1}+\sin \xi_{1} \sin \alpha_{1} \cos \varphi\right)^{2}}-c_{1} t\right)-i y\left(\sin \xi_{1} \cos \alpha_{1} \cos \varphi-\cos \xi_{1} \sin \alpha_{1}\right)+i x \sin \xi_{1} \sin \varphi}\right\} .
\end{aligned}
$$

In contrast to the incident and reflected cases, the transmitted azimuthally dependent pulses $\hat{\Psi}_{\varphi}(\mathbf{r}, t)$ have speeds that depend on $\varphi$. The square root coefficient of $z$ depends on $\cos \varphi$ and this dependence cannot be eliminated by a simple rotation of the coordinates. As a result of this dependence on $\varphi$, the transmitted azimuthally dependent pulses arrive at a certain observation point with different speeds. Except for normal incidence $\left(\alpha_{1}=0\right)$, these pulses do not arrive together at the same time to coherently construct an LW pulse. As a consequence, the transmitted pulse degenerates and its central peak decays as we move away from the surface of discontinuity.

The azimuthal angular representation of the transmitted field constitutes a framework that allows an accurate estimation of the localization depth of the transmitted field. Toward such a goal, we assume that the wave vectors of the trans- 
mitted spectral components form an approximate conical surface. The inclination angle of the axis of such a surface is $\alpha_{2}$ and its apex angle is $\xi_{2}$. Consider a section, taken at $x=0$, of the incident, reflected and transmitted cones, as illustrated in Fig. 2. Applying Snell's law to the rays shows that the following two equations are satisfied:

$$
\left(1 / c_{1}\right) \sin \left(\alpha_{1} \pm \xi_{1}\right)=\left(1 / c_{2}\right) \sin \left(\alpha_{2} \pm \xi_{2}\right) .
$$

The combination of these two equations yields the relations

$$
\begin{aligned}
& \left(1 / c_{1}\right) \sin \xi_{1} \cos \alpha_{1}=\left(1 / c_{2}\right) \sin \xi_{2} \cos \alpha_{2}, \\
& \left(1 / c_{1}\right) \cos \xi_{1} \sin \alpha_{1}=\left(1 / c_{2}\right) \cos \xi_{2} \sin \alpha_{2} .
\end{aligned}
$$

Eliminating the dependence on $\alpha_{2}$ and after some algebraic manipulation, we obtain

$$
\begin{aligned}
\cos ^{4} & \xi_{2}+\left(\left(c_{2} / c_{1}\right)^{2} \sin ^{2} \xi_{1} \cos ^{2} \alpha_{1}\right. \\
& \left.-\left(c_{2} / c_{1}\right)^{2} \cos ^{2} \xi_{1} \sin ^{2} \alpha_{1}-1\right) \cos ^{2} \xi_{2} \\
& +\left(c_{2} / c_{1}\right)^{2} \cos ^{2} \xi_{1} \sin ^{2} \alpha_{1}=0 .
\end{aligned}
$$

The solution to this equation gives the apex angle of the transmitted field; specifically,

$$
\cos \xi_{2}=\sqrt{-(B / 2)+\sqrt{(B / 2)^{2}-C}},
$$

where $\quad B=\left(\left(c_{2} / c_{1}\right)^{2} \sin ^{2} \xi_{1} \cos ^{2} \alpha_{1}-\left(c_{2} / c_{1}\right)^{2} \cos ^{2} \xi_{1} \sin ^{2} \alpha_{1}\right.$ -1) and $C=\left(c_{2} / c_{1}\right)^{2} \cos ^{2} \xi_{1} \sin ^{2} \alpha_{1}$. After evaluating the apex angle $\xi_{2}$, the inclination angle $\alpha_{2}$ can be calculated using either Eq. (33) or (34). This completes the analysis needed to calculate $\alpha_{2}$ and $\xi_{2}$. These values can be used to estimate the deviation of the square root coefficient of $z$ in Eq. (32b) from that of a rotated $z$ variable appearing in an exact X-wave characterized by $\xi_{2}$ and tilted by an angle $\alpha_{2}$. Toward such a goal, consider the denominators

$$
\begin{aligned}
D= & a+i\left(z \sqrt{\left(c_{1} / c_{2}\right)^{2}-1+\left(\cos \xi_{1} \cos \alpha_{1}+\sin \xi_{1} \sin \alpha_{1} \cos \varphi\right)^{2}}-c_{1} t\right) \\
& -i y\left(\sin \xi_{1} \cos \alpha_{1} \cos \varphi-\cos \xi_{1} \sin \alpha_{1}\right) \pm i x \sin \xi_{1} \sin \varphi
\end{aligned}
$$

that appear in Eq. (32b). Using Eqs. (33) and (34), they can be rewritten in terms of the rotated coordinates of the transmitted field $\left(x^{\prime}=x, y^{\prime}=y \cos \alpha_{2}-z \sin \alpha_{2}\right.$, and $\left.z^{\prime}=y \sin \alpha_{2}+z \cos \alpha_{2}\right)$ as follows:

$$
D=a+i\left(c_{1} / c_{2}\right)\left(z^{\prime} \cos \xi_{2}-c_{2} t\right)+i z\left(c_{1} / c_{2}\right) \Delta(\varphi)-i\left(c_{1} / c_{2}\right)\left(y^{\prime} \sin \xi_{2} \cos \varphi\right) \pm i x \sin \xi_{1} \sin \varphi .
$$

Here $\Delta(\varphi)$ is the deviation from the assumed trajectory specified by $\alpha_{2}$ and $\xi_{2}$ and is equal to

$$
\Delta(\varphi)=\sqrt{1-\left(c_{2} / c_{1}\right)^{2}+\left(c_{2} / c_{1}\right)^{2}\left(\cos \xi_{1} \cos \alpha_{1}+\sin \xi_{1} \sin \alpha_{1} \cos \varphi\right)^{2}}-\cos \xi_{2} \cos \alpha_{2}-\sin \xi_{2} \sin \alpha_{2} \cos \varphi .
$$

For short distances from the interface the condition $z\left(c_{1} / c_{2}\right) \Delta(\varphi) \ll a$ is satisfied for all values of $\Delta(\varphi)$. Hence, we introduce the hypothesis that the pulse stays localized with peak amplitude $\propto 1 / a$ until it reaches the following distance along the direction of propagation of the pulse:

$$
z_{d}^{\prime}=\frac{z_{d}}{\cos \alpha_{2}}=\frac{\left(c_{2} / c_{1}\right) a}{|\bar{\Delta}(\varphi)| \cos \alpha_{2}}
$$

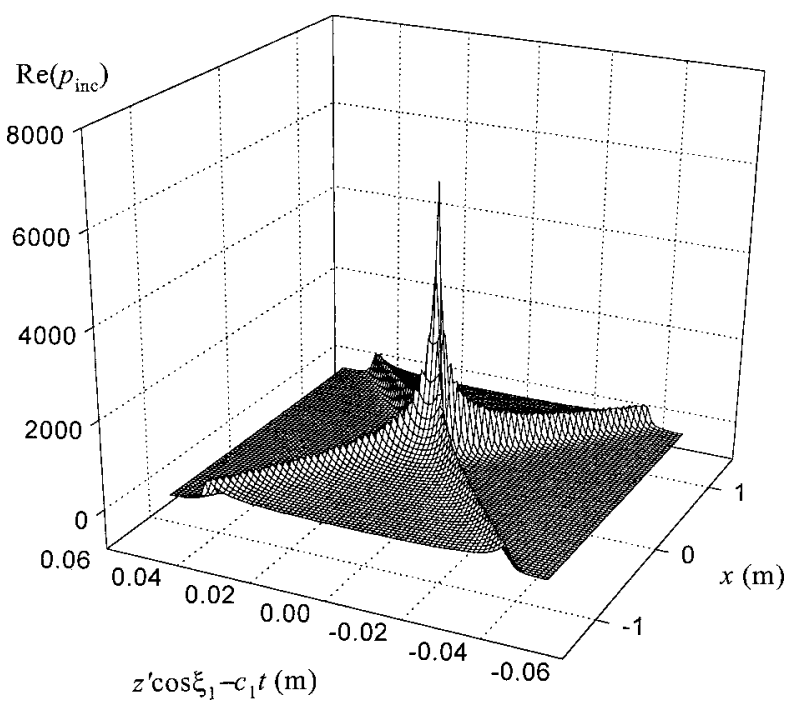

FIG. 3. Pressure field of the incident X-wave having $a=0.001 \mathrm{~m}$ and $\xi_{1}$ $=2^{\circ}$ evaluated at $z=-1 \mathrm{~m}$.
Here, $\bar{\Delta}(\varphi)$ is the average deviation over the range $0 \leqslant \varphi<\pi$. From Eq. (40) it appears that $z_{d}$ increases with $\left(c_{2} / c_{1}\right)$; however, $|\bar{\Delta}(\varphi)| \propto\left(c_{2} / c_{1}\right)^{n}(n>1)$, resulting in $z_{d}$ being inversely proportional to $\left(c_{2} / c_{1}\right)$. The dispersion-free range given in Eq. (40) is a fairly accurate estimate as will be seen from the examples in the next section.

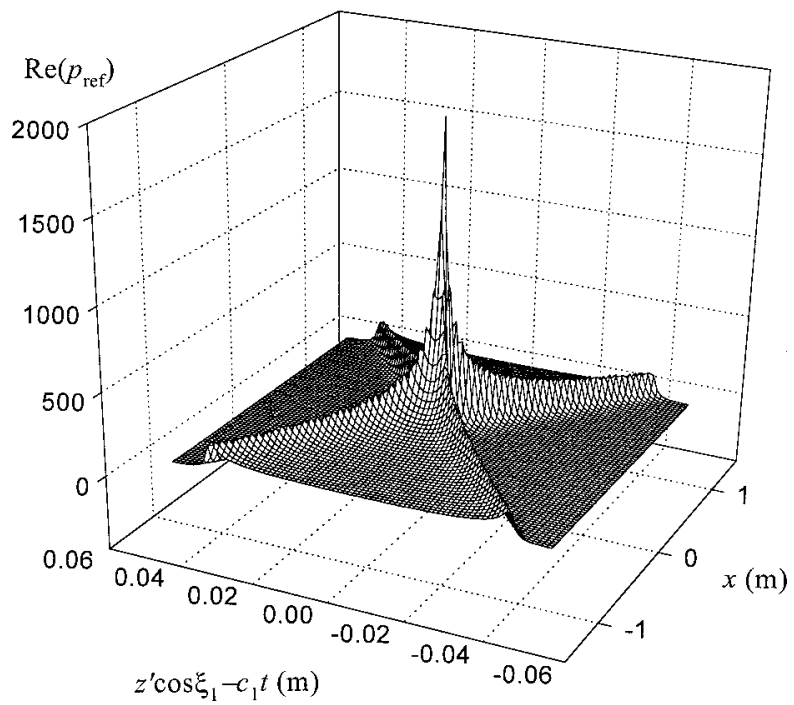

FIG. 4. Reflected pressure field evaluated for $\left(c_{1} / c_{2}\right)=2$ and $\left(\rho_{01} / \rho_{02}\right)$ $=0.25$. The incident $\mathrm{X}$-wave has a propagation-axis tilted at $\alpha_{1}=25^{\circ}$. The surface plot is calculated at $z=-1 \mathrm{~m}$. 


\section{ILLUSTRATIONS AND EXAMPLES}

In this section, we study the properties of the reflected and transmitted pressure fields resulting from an X-wave incident on a planar interface. The azimuthal angular representation introduced in the preceding section is used to numerically calculate surface plots of the reflected and transmitted pulses. The aim of the examples employed is to highlight certain properties of the reflected or transmitted fields and to illustrate that the disintegration of the transmitted field is caused by the spectral incoherence described in Sec. III. As such, the examples used will demonstrate the validity of the expression deduced for the dispersion-free length given in Eq. (40). Two main situations are considered; namely, the incident $\mathrm{X}$-wave approaches the interface either from the side of the faster medium $\left[\left(c_{1} / c_{2}\right)>1\right]$ or the slower one $\left[\left(c_{1} / c_{2}\right)<1\right]$.

\section{A. Reflected and transmitted fields for $\left(c_{1} / c_{2}\right)>1$}

The X-wave is assumed to approach the surface of discontinuity from the side of the medium having the larger wave velocity; specifically, the values $\left(c_{1} / c_{2}\right)^{2}=4$ and $\left(\rho_{01} / \rho_{02}\right)=0.25$ are chosen. In most numerical results presented in this section, the $\mathrm{X}$-wave shown in Fig. 3 is assumed to be incident on the interface at an inclination angle $\alpha_{1}$ $=25^{\circ}$ and is characterized by the parameters $\xi_{1}=2^{\circ}$ and $a$ $=0.001 \mathrm{~m}$, except when explicitly stated otherwise. Furthermore, the calculated amplitudes of all incident, reflected and transmitted fields are displayed in terms of $\left(A_{0} / 2\right)$. The timevariation of the reflected pressure field is plotted in Fig. 4 for $y^{\prime}=0, z=-1 \mathrm{~m}$. The reflected X-wave retains the same LW structure characterizing the incident pressure field. At farther distances from the interface the reflected pulse preserves its shape and does not spread out.

As discussed in the preceding section, the refracted pulse should start disintegrating after traversing the estimated dispersion-free range given in Eq. (40). To illustrate this point the transmitted pressure field is plotted in Fig. 5 as a function of the transverse variable $x\left(y^{\prime}=0\right)$ at distances $z=0.1,50$, and $250 \mathrm{~m}$ from the interface. The time dependence of the field is evaluated along the tilted axis of propagation as a function of the quantity $z^{\prime} \cos \xi_{2}-c_{2} t$. For an $\mathrm{X}$-wave pulse having $\xi_{1}=2^{\circ}$ and $a=0.001 \mathrm{~m}$ incident at an angle $\alpha_{1}=25^{\circ}$ on an interface separating two media characterized by $\left(c_{1} / c_{2}\right)^{2}=4$ and $\left(\rho_{01} / \rho_{02}\right)=0.25$, the dispersionfree range given in Eq. (40) yields $z_{d}=45.77 \mathrm{~m}$. This result agrees nicely with the decay pattern illustrated in Fig. 5. The transmitted $\mathrm{X}$-wave retains its shape for short distances, while the peak starts decaying at a distance of about $50 \mathrm{~m}$. It appears, from the figures, that the disintegration of the transmitted field is accompanied by an increase in the axial width of the central portion of the pulse. This behavior is related to the dispersion in the velocities of the azimuthally dependent pulsed components. The disintegration of the pulse starts when the spread in the arrival time of the azimuthally dependent pulses is larger than $a / c_{2}$.

To achieve a better understanding of the decay properties of refracted $\mathrm{X}$-wave, we consider the effect of changing the inclination angle $\alpha_{1}$, the axicon angle $\xi_{1}$ and the param-
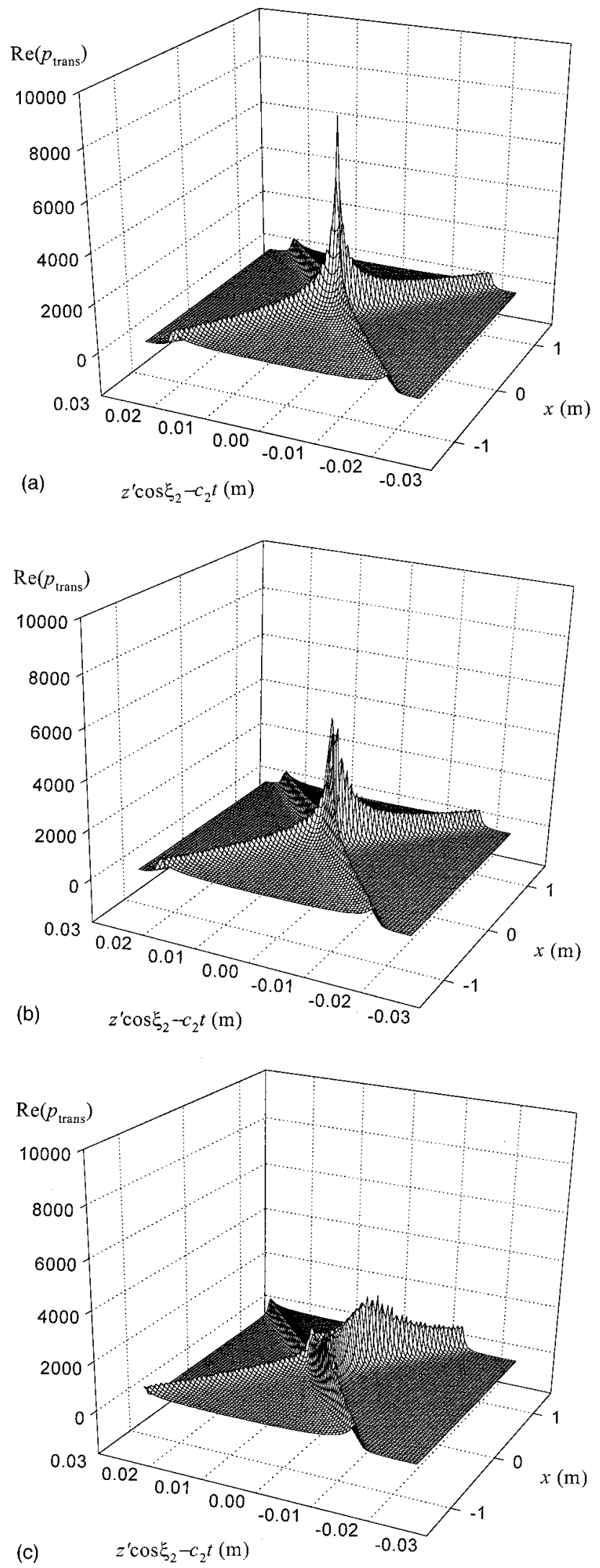

FIG. 5. Transmitted pressure field evaluated for $\left(c_{1} / c_{2}\right)=2$ and $\left(\rho_{01} / \rho_{02}\right)$ $=0.25$. The incident $\mathrm{X}$-wave has a propagation-axis tilted at $\alpha_{1}=25^{\circ}$. The surface plots are calculated at (a) $z=0.1 \mathrm{~m}$, (b) $z=50 \mathrm{~m}$ and (c) $z$ $=250 \mathrm{~m}$ 

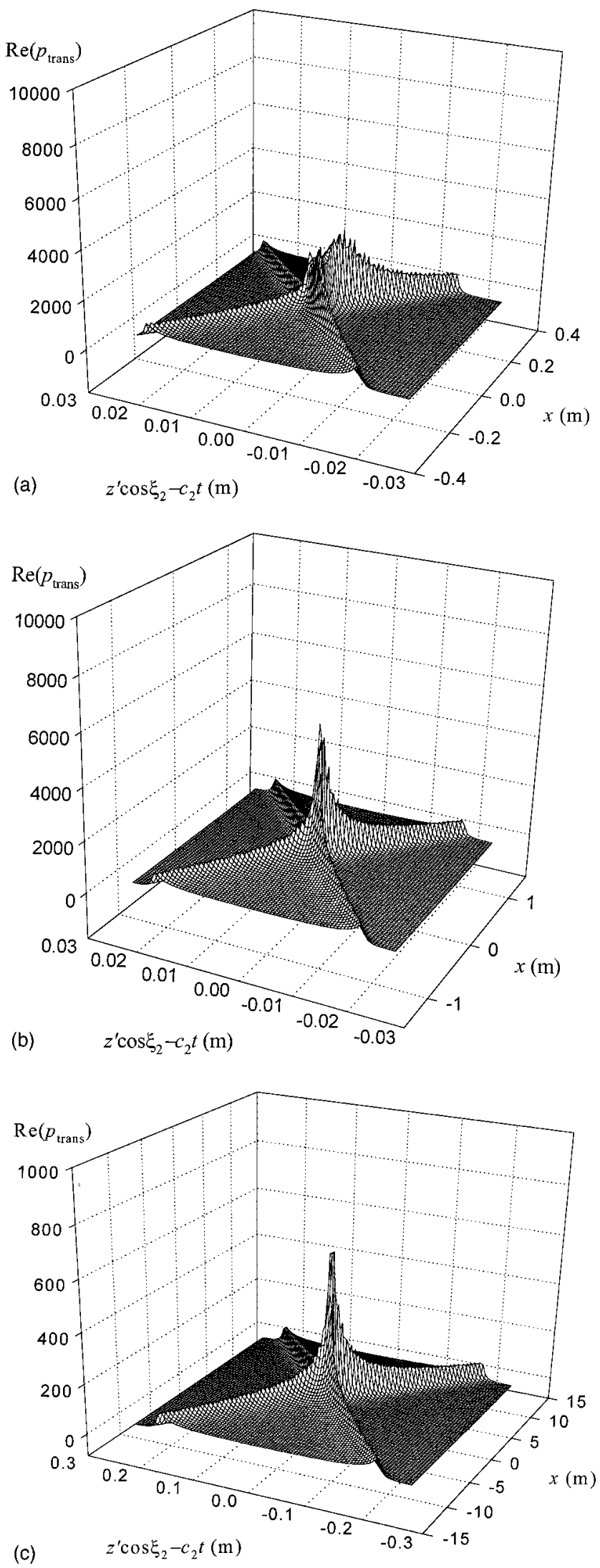

FIG. 6. Transmitted pressure field evaluated at $z=10 \mathrm{~m}$ showing the change in the dispersion rate when (a) the apex angle $\xi_{1}$ is increased to $8^{\circ}$ and (b) the inclination angle $\alpha_{1}=50^{\circ}$. (c) The transmitted pressure field evaluated at $z=250 \mathrm{~m}$ when the parameter $a$ increases to $0.01 \mathrm{~m}$. In each case the rest of the parameters are unchanged and are the same as in Fig. 5. eter $a$ on the dispersion-free range $z_{d}$. In Fig. 6(a), it is seen that increasing $\xi_{1}$ from $2^{\circ}$ to $8^{\circ}$ causes the transmitted pulse to decay at a much faster rate. The figure shows that for $\xi_{1}$ $=8^{\circ}$ the peak of the pulse has already decayed significantly at $z=10 \mathrm{~m}$. The resulting decay is a good demonstration of the fact that changing the apex angle to $\xi_{1}=8^{\circ}$, while keeping the other parameters unchanged, reduces the dispersionfree depth to the value $z_{d}=2.86 \mathrm{~m}$. On the other hand, changing the inclination angle $\alpha_{1}$ from $25^{\circ}$ to $50^{\circ}$, while keeping $\xi_{1}=2^{\circ}$, yields $z_{d}=11.76 \mathrm{~m}$. Figure $6(\mathrm{~b})$ confirms this change in the dispersion-free length by demonstrating that increasing the inclination angle to $\alpha_{1}=50^{\circ}$ causes the peak of the transmitted pulse to start decaying at $z=10 \mathrm{~m}$. Figure 6(c) shows the transmitted field at $z=250 \mathrm{~m}$ for $a$ $=0.01 \mathrm{~m}$. This agrees with the fact that increasing the parameter $a$ to $0.01 \mathrm{~m}$, while keeping $\alpha_{1}$ and $\xi_{1}$ unchanged, extends the localization range to $z_{d}=457.73 \mathrm{~m}$. It is interesting to point out that parameter $a$ controls the temporal frequency bandwidth. ${ }^{20}$ As $a$ increases, the bandwidth decreases. Thus $\mathrm{X}$-waves with smaller frequency bandwidth undergo less dispersion, as they travel inside the second material.

\section{B. Reflected and transmitted fields for $\left(c_{1} / c_{2}\right)<1$}

In this subsection, the analysis introduced in Sec. III is applied to the case of an X-wave incident from the slower medium; specifically, we assume that $\left(c_{1} / c_{2}\right)^{2}=0.25$ and $\left(\rho_{01} / \rho_{02}\right)=4$. The field components incident at spectral angles $\theta_{k}>\theta_{c}=\sin ^{-1}\left(c_{1} / c_{2}\right)$ will be totally reflected and evanescent fields are generated inside the second medium. In this section, we investigate two cases involving the evanescent fields, associated with normally and obliquely incident $\mathrm{X}$-waves, respectively. For the former case, the evanescent fields are generated when the axicon angle of the incident $\mathrm{X}$-wave $\xi_{1}>\theta_{c}=\sin ^{-1}\left(c_{1} / c_{2}\right)$. Consequently, the $\mathrm{X}$-wave is totally reflected and all the spectral components produce evanescent fields in the second medium. For this particular case, the evanescent fields can be deduced in an exact closed-form. This allows for an interesting assessment of the time development of the evanescent field. For oblique incidence several possible situations are investigated, namely sub-critical, super-critical and near-critical incidence. For the first case, all the spectral components have $\theta_{k}<\sin ^{-1}\left(c_{1} / c_{2}\right)$. This is completely analogous to the case discussed in Sec. III, except that the disintegration should be faster as seen from Eq. (40). The super-critical case corresponds to the situation when all the wave vectors lying on the tilted spectral cone are inclined at angles $\theta_{k}>\sin ^{-1}\left(c_{1} / c_{2}\right)$. Near-critical incidence involves the situation when the tilted spectral cone has some wave vectors that are tilted at $\theta_{k}<\sin ^{-1}\left(c_{1} / c_{2}\right)$, while the rest of the wave vectors have incidence angles greater than the critical angle. The field transmitted into the second medium is thus a mixture of propagating and evanescent fields. Near the interface, the lateral waves and the refracted ones become concentrated in a small region, thus producing a highly compressed and focused pulse.

Starting with normal incidence, we assume that the incident $\mathrm{X}$-wave is characterized by an axicon angle $\xi_{1}=40^{\circ}$ 
$>\sin ^{-1}(1 / 2)=30^{\circ}$. The transmitted field follows directly from Eqs. (26a) and (26b), where for normal incidence $\alpha_{1}$ $=0$ and $\eta_{1}=k_{y}$, thus yielding

$$
\begin{aligned}
P_{\text {trans }}(\mathbf{r}, t)= & \int_{0}^{\infty} d\left(\omega / c_{1}\right) \int_{-\omega / c_{1} \sin \xi}^{\omega / c_{1} \sin \xi} d k_{y} \frac{A_{0} e^{-\left(\omega / c_{1}\right) a}}{\sqrt{\left(\omega / c_{1}\right)^{2} \sin ^{2} \xi_{1}-k_{y}^{2}}} \\
& \times \cos \left(x \sqrt{\left(\omega / c_{1}\right)^{2} \sin ^{2} \xi_{1}-k_{y}^{2}}\right) \\
& \times e^{i \omega t} e^{-i k_{y} y} e^{-z\left(\omega / c_{1}\right) \sqrt{\sin ^{2} \xi_{1}-\left(c_{1} / c_{2}\right)^{2}}} T\left(\xi_{1}\right),
\end{aligned}
$$

where

$$
T\left(\xi_{1}\right)=\frac{2 \cos \xi_{1}\left(c_{1} / c_{2}\right)^{2}}{\cos \xi_{1}\left(c_{1} / c_{2}\right)^{2}-i \sqrt{\sin ^{2} \xi_{1}-\left(c_{1} / c_{2}\right)^{2}}} .
$$

In the above expression, we have used the negative imaginary branch for which $\sqrt{\left(\omega / c_{2}\right)^{2}-\left(\omega / c_{1}\right)^{2} \sin ^{2} \xi_{1}}$ $=-i\left(\omega / c_{1}\right) \sqrt{\sin ^{2} \xi_{1}-\left(c_{1} / c_{2}\right)^{2}}$. This choice yields an exponentially decaying dependence on $z$ in the integrand of Eq. (41a). In addition, we have assumed that $\left(\rho_{01} / \rho_{02}\right)$ $=\left(c_{2} / c_{1}\right)^{2}$ in order to simplify the resulting mathematical expressions. Since $T\left(\xi_{1}\right)$ is independent of $\left(\omega / c_{1}\right)$ and $k_{y}$, the double integration in Eq. (41a) can be evaluated exactly to give

$$
p_{\text {trans }}(\mathbf{r}, t)=\frac{\left(2 A_{0} \cos \xi_{1}\left(c_{1} / c_{2}\right)^{2}\right) /\left(\cos \xi_{1}\left(c_{1} / c_{2}\right)^{2}-i \sqrt{\sin ^{2} \xi_{1}-\left(c_{1} / c_{2}\right)^{2}}\right)}{\sqrt{\rho^{2} \sin ^{2} \xi_{1}+\left(a+z \sqrt{\sin ^{2} \xi_{1}-\left(c_{1} / c_{2}\right)^{2}}-i c t\right)^{2}}} .
$$

This evanescent field exhibits a transverse wave motion along the interface through the terms $\rho^{2} \sin ^{2} \xi_{1}-\left(c_{1} t\right)^{2}$ appearing in the square root in the denominator. This transverse motion is illustrated in Fig. 7 where surface plots of the real part of $p_{\text {trans }}(\mathbf{r}, t)$ are shown for different times as a function of $z$, the normal distance from the interface. The two localized bumps that appear in Fig. 7(a) approach each other for negative time and coalesce into a single peak at $c_{1} t \approx 0$, as shown in Fig. 7(b). Henceforth, they split into two bumps and travel away from each other for $c_{1} t>0$ [cf. Fig. 7(c)]. The time evolution of the evanescent field is illustrated in Fig. 8(a). A surface plot of the real part of $p_{\text {trans }}(\mathbf{r}, t)$ given in Eq. (42) is illustrated at distance $z=0.001 \mathrm{~m}$. At the farther distances, the evanescent field has the same shape but spreads out and its amplitude decreases. The time dependence of the evanescent field near the surface is an attenuated imprint of the reflected field [cf. Fig. 8(b)]. The reflected pulse could, thus, be perceived to exist for a certain period of time inside the second medium before being reflected back into the first one. This is analogous to the quantum mechanical picture that describes the state of a totally reflected wave packet from a finite-height potential barrier. In the latter situation, the wave packet dwells inside the reflecting barrier for a short time before being totally reflected. In both cases, the spectral components of the reflected fields undergo phase delays that distort the shape of the reflected pulses. Such phase delays are responsible for the distortion of the X-shape of the reflected field shown in Fig. 8(b). However, the reflected pulse preserves its shape from this time on and does not undergo any dispersion as it travels away from the interface.

To gain a better understanding of the time evolution of the evanescent field, we consider the case when $\rho=0$. As a consequence, the real part of the expression given in Eq. (42) acquires the following simple form:

$$
\operatorname{Re}\left\{p_{\text {trans }}(\mathbf{r}, t)\right\}=\frac{A_{0}\left(2 a \cos ^{2} \xi_{1}\left(c_{1} / c_{2}\right)^{2}+2 \cos \xi_{1}\left(c_{1} / c_{2}\right)^{2} \sqrt{\sin ^{2} \xi_{1}-\left(c_{1} / c_{2}\right)^{2}}\left(\left(c_{1} / c_{2}\right)^{2} \cos \xi_{1} z-c_{1} t\right)\right)}{\left(\left(c_{1} t\right)^{2}+\left(a+z \sqrt{\sin ^{2} \xi_{1}-\left(c_{1} / c_{2}\right)^{2}}\right)^{2}\right)\left(\left(c_{1} / c_{2}\right)^{4} \cos ^{2} \xi_{1}+\sin ^{2} \xi_{1}-\left(c_{1} / c_{2}\right)^{2}\right)} .
$$

As shown in Fig. 8, this field has two peaks of opposite signs with a null in between. The null travels at the velocity $\mathrm{v}_{\text {evan }}=c_{2} /\left(c_{1} / c_{2}\right) \cos \xi_{1}$ greater than the velocity $c_{2}$ of the medium. This explicit propagation in the $z$ direction of the evanescent field is an interesting result in its own right. Thus in addition to the lateral wave motion of the evanescent field there is a longitudinal transfer of the field in a direction normal to the interface. This tunnelinglike effect may be used for ultra-fast signaling through a thin slab using evanescent fields.

For oblique incidence, we consider an incident $\mathrm{X}$-wave characterized by an axicon angle $\xi_{1}=2^{\circ}$. The axis of propa- gation of the $\mathrm{X}$-wave is inclined at angles $\alpha_{1}=15^{\circ}, 30^{\circ}$ and $45^{\circ}$, corresponding to sub-critical, near-critical and supercritical incidence, respectively. The angular superposition given in Eq. (32) is used to evaluate the transmitted fields in the above three cases, while Eq. (30) is employed to calculate the reflected fields. In these two equations, one should be careful when the argument of $\sqrt{\left(c_{1} / c_{2}\right)^{2}-1+\left(\cos \xi_{1} \cos \alpha_{1}+\sin \xi_{1} \sin \alpha_{1} \cos \varphi\right)^{2}}$ becomes negative. In such a case, the negative imaginary branch must be chosen to ensure the decay of the evanescent field along the positive $z$ direction.

For sub-critical incidence, we assume that the $\mathrm{X}$-wave 

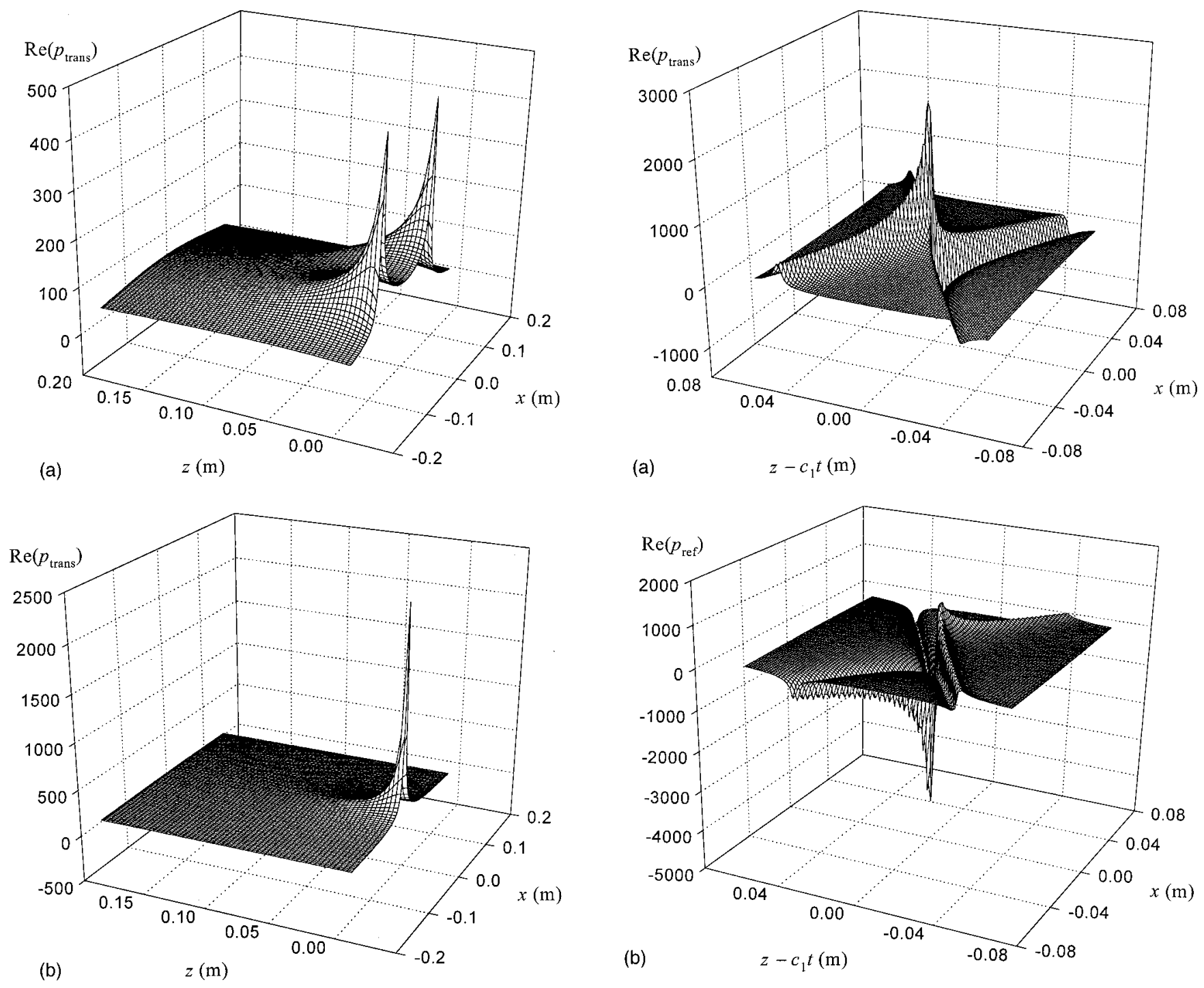

FIG. 8. (a) Time dependence of the evanescent fields resulting from a normally incident X-wave having $a=0.001 \mathrm{~m}$ and $\xi_{1}=40^{\circ}$. The surface plots are evaluated for $\left(c_{1} / c_{2}\right)=0.5$ at $z=0.001 \mathrm{~m}$. (b) The totally reflected pressure field evaluated at $z=-1 \mathrm{~m}$.

plotted in Fig. 3 is incident at an angle $\alpha_{1}=15^{\circ}$ on the interface separating two media having $\left(c_{1} / c_{2}\right)=0.5$. The reflected pressure field calculated at $z=-1$ is shown in Fig. 9(a). The reflected pulse resembles the incident X-wave except for a change in the sign and magnitude of its amplitude. In Fig. 9(b)-(d), the transmitted field is plotted for distances $z=0.1,1$ and $50 \mathrm{~m}$ normal to the interface. One should note that the decay of the peak of the transmitted field is much faster than in the case $\left(c_{1} / c_{2}\right)=2$. The pulse has already started to disperse at $z=1 \mathrm{~m}$ and has decayed significantly at $z=50 \mathrm{~m}$. Another interesting difference between the two cases $\left(c_{1} / c_{2}\right)>1$ and $\left(c_{1} / c_{2}\right)<1$ is that in the former the pulse spreads backward along the lagging X-shaped arms, while in the latter the transmitted pulse spreads out along the forward X-shaped arms. This behavior is due to the fact that the wave vectors of the transmitted field lie on a convoluted conic surface. ${ }^{31}$ Such a deformed cone is shaped by stretching the apex point of the cone associated with the incident $\mathrm{X}$-wave into a line segment. For $\left(c_{1} / c_{2}\right)>1$, the apex point is drawn outward to form a line segment in a direction re-

FIG. 7. Evanescent fields resulting from a normally incident $X$-wave having $a=0.001 \mathrm{~m}$ and $\xi_{1}=40^{\circ}$. The surface plots are evaluated for $\left(c_{1} / c_{2}\right)$ $=0.5$ at (a) $c_{1} t=-0.05 \mathrm{~m}$, (b) $c_{1} t=0$ and (c) $c_{1} t=0.05 \mathrm{~m}$. 

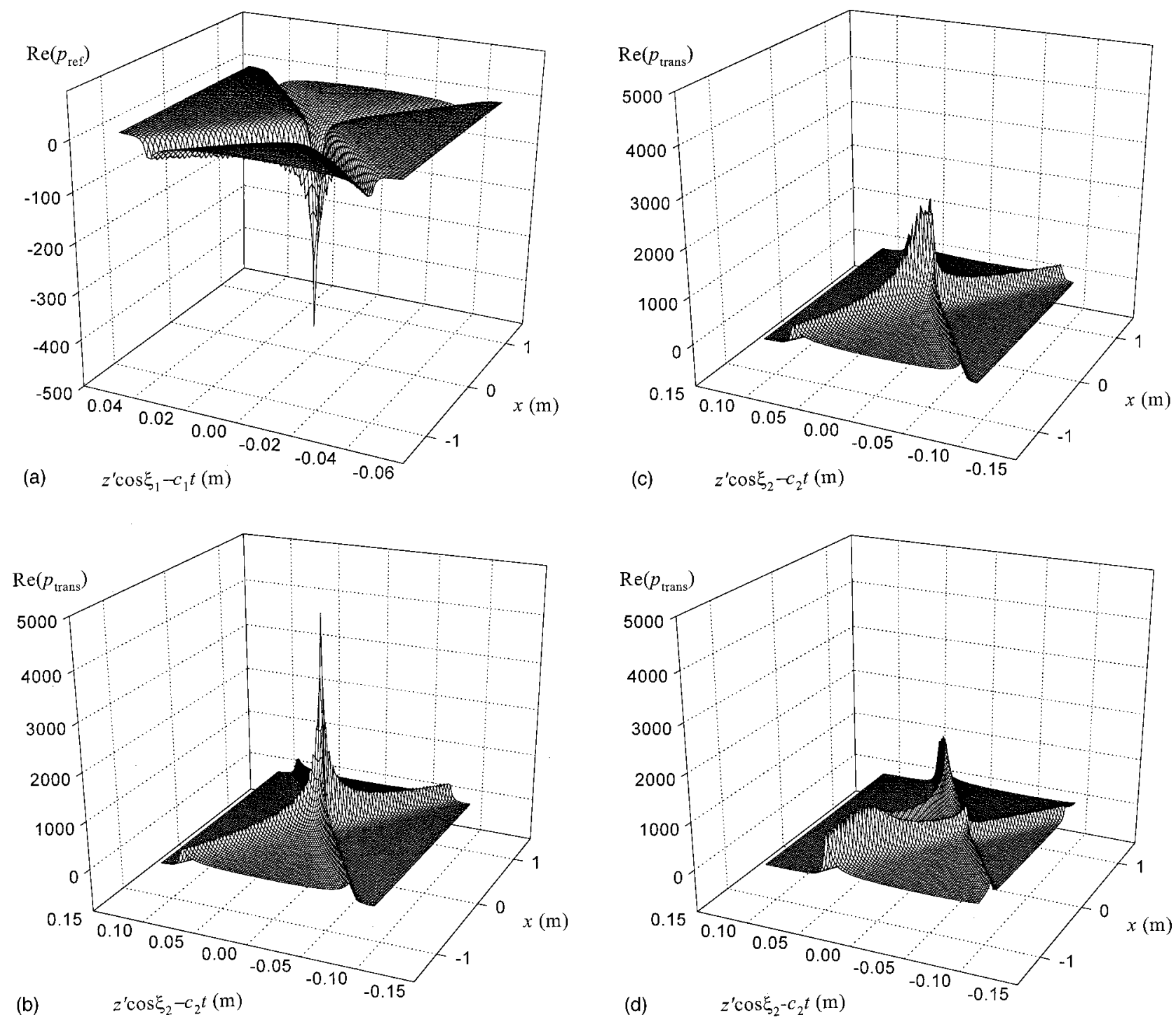

FIG. 9. Sub-critical incidence at $\alpha_{1}=15^{\circ}<\theta_{c}=30^{\circ}\left(\left(c_{1} / c_{2}\right)=0.5\right)$. (a) Reflected pressure field plotted at $z=-1 \mathrm{~m}$. Transmitted pressure field is evaluated at (b) $z=0.1 \mathrm{~m}$, (c) $z=1 \mathrm{~m}$ and (d) $z=50 \mathrm{~m}$.

moved away from the interface. Wave components arriving at different points on this line segment lag behind the peak. When $\left(c_{1} / c_{2}\right)<1$, the apex is stretched inward toward the interface forming a folded surface. Wave components contributing to the transmitted field lead over the peak. Details of these ideas are presented in a separate work (cf. Ref. 31).

For the case of super-critical incidence, the pulse shown in Fig. 3 is incident on the surface of discontinuity at an angle $\alpha_{1}=45^{\circ}$. All the plane wave components of the incident pressure field are inclined at angles $\theta_{k}$ larger than the critical angle $\theta_{c}=30^{\circ}$. Only evanescent fields exist in the second medium. The properties of the totally reflected field and the evanescent fields are similar to those discussed above for normal incidence with $\xi_{1}>\theta_{c}$. To verify our claim, we provide in Fig. 10 a plot of the time variation of the totally reflected pressure field at $z=-1 \mathrm{~m}$. The reflected pulse has an LW structure and does not disperse as it travels away from the interface. The evanescent field dependence on $z$, the perpendicular distance to the interface, is similar to the case of normal incidence. In analogy to Fig. 7, the two lateral

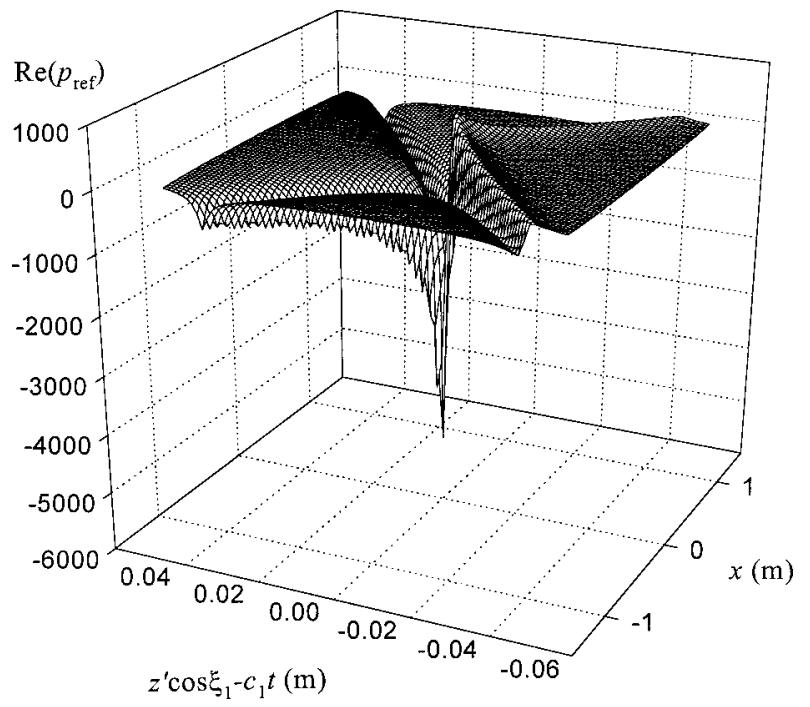

FIG. 10. Reflected pressure field plotted at $z=-1 \mathrm{~m}$ for super-critical incidence at $\alpha_{1}=45^{\circ}>\theta_{c}=30^{\circ}\left[\left(c_{1} / c_{2}\right)=0.5\right]$. 
peaks of the evanescent field approach each other before coalescing at $c_{1} t \approx 0$ and then start traveling away from each other.

Near-critical incidence corresponds to situations when the inclination angles of the wave vectors associated with the spectral components of the incident pressure field can have both values $\theta_{k}>\theta_{c}$ and $\theta_{k}<\theta_{c}$. The spectral components having $\theta_{k}<\theta_{c}$ generate propagating refracted fields in the second medium, while those having $\theta_{k}>\theta_{c}$ produce evanescent fields. The transmitted field, thus, consists of a combination of these two types of waves. Various transmitted field distributions are evaluated for different $\alpha_{1}$ angles in the vicinity of $\theta_{c}=30^{\circ}$. This allows us to acquire a better understanding of the behavior of the transmitted mixture of refracted and evanescent fields. In Fig. 11 we plot the transmitted pressure fields resulting from an $\mathrm{X}$-wave pulse (cf. the pulse shown in Fig. 3, having $\xi_{1}=2^{\circ}$ and $a$ $=0.001 \mathrm{~m}$ ) incident at angles $\alpha_{1}=28^{\circ}, 29^{\circ}, 30^{\circ}, 31^{\circ}$ and $32^{\circ}$. The transmitted fields, shown in Fig. 11(a) and (e), are similar to those occurring for sub-critical and super-critical incidence, respectively. This is the case because for $\xi_{1}$ $=2^{\circ}$, the pulse plotted in Fig. 11(a) is transmitted due to the incidence of an X-wave consisting of spectral components having $\theta_{k}<\theta_{c}$. The opposite is true for the pulse shown in Fig. 11(e). The transmitted field due to an $\mathrm{X}$-wave inclined at $\alpha_{1}=31^{\circ}$ is plotted in Fig. 11(d) and shows a close resemblance to that corresponding to $\alpha_{1}=32^{\circ}$ shown in Fig. 11(e). The reason for this resemblance is that, for $\alpha_{1}=31^{\circ}$, most of the spectral components have $\theta_{k}>\theta_{c}$. One should note, however, that the transmitted pulse shown in Fig. 11(d) is slightly compressed along the direction of propagation and has larger amplitude than the pulse plotted in Fig. 11(e). Far away from the interface, refracted components of the pulse shown in Fig. 11(d) will be stripped away from the decaying evanescent components, forming a propagating pulse that disperses with distance. The pulse shown in Fig. 11(e), however, consists solely of evanescent fields that will have negligibly small amplitudes at farther distances from the interface.

The pressure field displayed in Fig. 11(b) shows an extremely strong pulse-compression effect. It appears that the planar interface acts as a lens that focuses and compresses the field transmitted into the second medium. This effect can be used in applications requiring the delivery of high intensity ultra-short pulses near an interface. This pulse focusing effect may be understood by resorting to the ray-theoretic approach introduced in Ref. 31. For $\left(c_{1} / c_{2}\right)<1$, the apex of the transmitted cone is stretched inwards into a line segment. This line segment is dragged up in the direction of the interface. For near-critical incidence, the line segment reaches the interface. Spectral components having $\theta_{k}<\theta_{c}$ will have refracted wave vectors ending up on different points of the line segment, while components with $\theta_{k}>\theta_{c}$ will produce lateral rays parallel to the interface. The concentration of such wave components in a small volume produces a highly focused pulse. At this point, we show that after traveling a short distance farther away from the interface, the amplitude of the transmitted field undergoes a sign inversion. In Fig. 12, we show that at $z=0.00109 \mathrm{~m}$ the transmitted pulse acquires very large negative amplitudes and is highly compressed. The extent of the compression of the pulse at $z$ $=0.00109 \mathrm{~m}$ can be emphasized further by comparing Figs. 11(b) and 12. The scale of the transverse variable is reduced by 10 -fold, while the longitudinal scale is shrunk by 20 -fold. The peak amplitude of the transmitted pulse is 200 times larger than the incident X-wave. This compression effect is not restricted only to the angle $\alpha_{1}=29^{\circ}$ but can occur for other angles at near-critical incidence. However, the position of the focus point varies with the various combinations of refracted and lateral rays. Consequently, incidence at different angles $\alpha_{1} \sim \theta_{c}$ results in pulse compression at different distances from the interface. In fact, a surface plot of the transmitted pulse, displayed in Fig. 11(c) for $\alpha_{1}=30^{\circ}$, shows that the inverted pulse is already focused at $z$ $=0.001 \mathrm{~m}$. This means that the strong focusing of the pulse occurs nearer to interface because the refracted rays are crowded together at a shorter distance from the surface of discontinuity. Furthermore, the field incident at $\alpha_{1}=30^{\circ}$ contains spectral components having $\theta_{k}>\theta_{c}$ that are totally reflected. Since a number of the spectral components are totally reflected while the remaining ones give rise to both reflected and refracted fields, we expect the reflected pressure field to lose its X-shaped distribution. This effect is illustrated in Fig. 13 where the reflected field corresponding to $\alpha_{1}=30^{\circ}$ is plotted at $z=-1 \mathrm{~m}$. One should note the deformation of the lagging $\mathrm{X}$-shaped arms. However, this pulse still has an LW structure and it travels away from the interface without any dispersion.

\section{CONCLUSION}

In this work, the reflection and transmission of acoustic $\mathrm{X}$-waves obliquely incident on a planar interface has been investigated. The analysis adopted is based on a spectral decomposition of the incident pulse into plane wave components specified by wave vectors forming a conical surface. The boundary conditions at the interface are satisfied by the individual plane waves and the reflected and transmitted fields are deduced as Fourier superpositions. Subsequently, it has been found profitable to transform each of these Fourier superpositions into an angular integration (synthesis) of azimuthally dependent pulsed plane waves. A study of the constituent pulsed solutions has been carried out for the problem under investigation in this paper. It has been shown that the incident and reflected pressure waves have azimuthally dependent pulses traveling at the same speed and, consequently, adding up coherently at any observation point to form an LW pulse. In contradistinction, the azimuthally dependent pulses associated with the transmitted field travel at different speeds, thus causing the dispersion of an LW pulse after traveling a certain distance from the interface. It has been shown that the localization range of the transmitted field depends on the temporal frequency bandwidth, the angle of inclination and the axicon angle of the incident $\mathrm{X}$-wave pulse. The main factor affecting the disintegration of the transmitted pulse is the axial width of the incident $\mathrm{X}$-wave along the direction of propagation (i.e., for $x^{\prime}=y^{\prime}$ $=0)$. For larger axial widths of the incident $\mathrm{X}$-wave, the transmitted pulse suffers less disintegration as it travels in- 

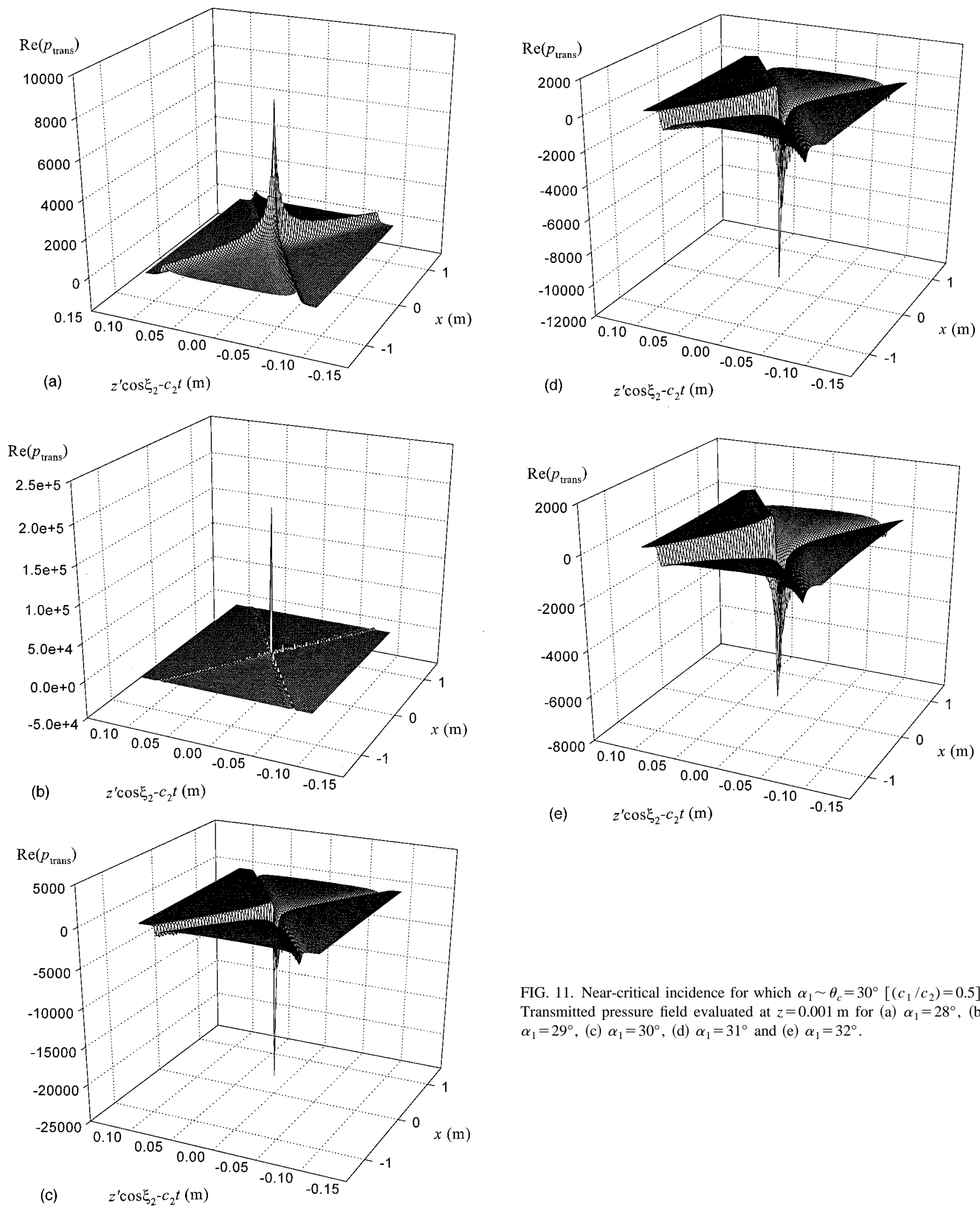

side the second medium. For zero angle of inclination (normal incidence), the transmitted fields retain their LW structure. These results are in agreement with the conclusions inferred from the ray-theoretic approach introduced in Ref. 31. The use of such angular superposition constitutes an efficient method for deducing the exact form of the transmitted field and for estimating its range of localization. 


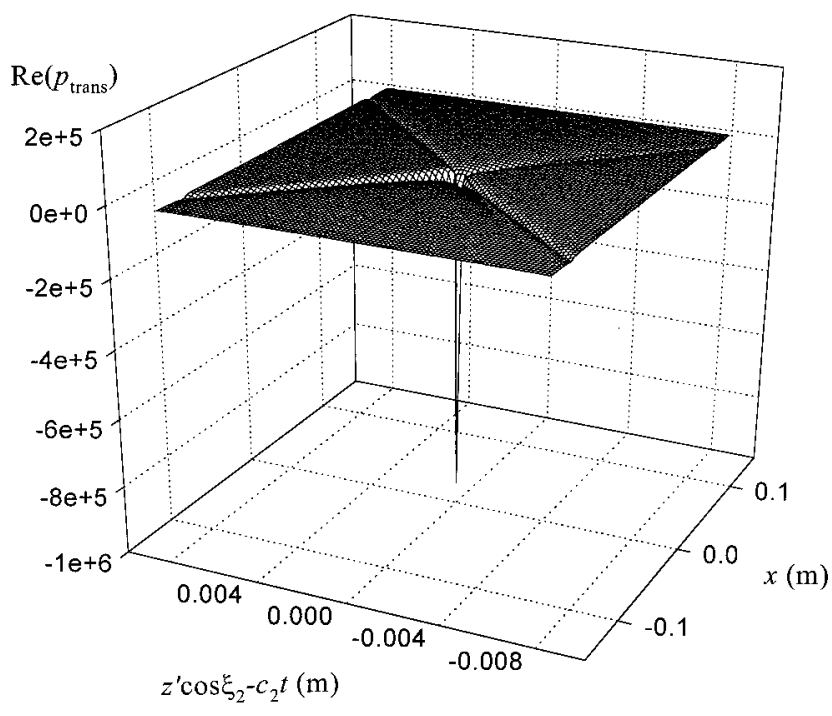

FIG. 12. Transmitted pressure field evaluated at $z=0.00109 \mathrm{~m}$ for $\alpha_{1}$ $=29^{\circ} ;\left(c_{1} / c_{2}\right)=0.5$.

dispersion of the transmitted pulse and the distance at which it starts. Such information is essential for practical applications that make use of the localized character of the transmitted field; e.g., in detection and identification of buried objects. The information should be used in conjunction with the fact that finite-energy LWs have a finite range of localization. Thus one should always be able to determine which effect will be the earliest to cause the decay of the transmitted field, the diffraction effect due to the initial finite energy source or the dispersion effect described in this paper. Such an assessment can be effected by comparing the estimate for the range of localization $z_{d}^{\prime}$ given in Eq. (40) to formulas specifying the diffraction lengths of LWs. ${ }^{6,14,20,32,33}$

Finally, one should note that we have basically studied the reflection and transmission of the infinite energy X-wave. As discussed earlier, the exact X-wave can be perceived as an interference of pulsed plane waves propagating along a conical surface and all having the same conical angle $\xi$ with

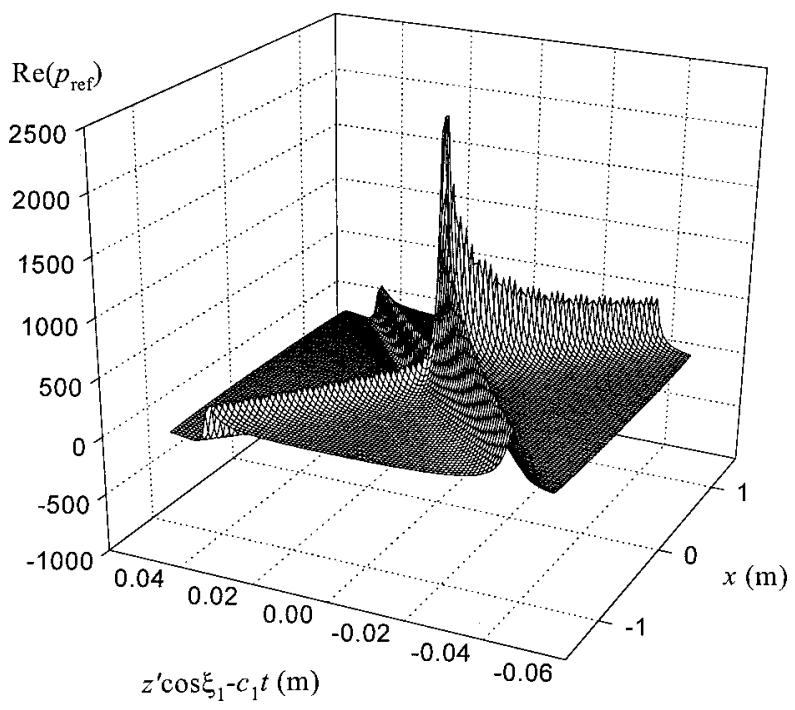

FIG. 13. Reflected pressure field evaluated at $z=-1 \mathrm{~m}$ for $\alpha_{1}=30^{\circ}$; $\left(c_{1} / c_{2}\right)=0.5$. respect to the axis of propagation. ${ }^{31}$ The resulting field has a slow radial decay and similarly to FWMs and to plane wave solution, X-waves have infinite energies. Finite energy $\mathrm{X}$-shaped solutions can be obtained either by truncating the transverse support of the field of the X-wave s,6,15,20 $^{5}$ or by using superpositions of superluminally boosted elementary solutions. ${ }^{21}$ Evidently, finite energy $\mathrm{X}$-waves are fields of interest in physical situations. However, the mathematical analysis needed to study the reflection and transmission of such pulses can be quite tedious. This is the reason for considering the canonical problem analyzed in this work. The results of this paper are thus useful in situations when the distances traveled by the finite energy $\mathrm{X}$-waves are smaller than their diffraction-free ranges. In addition, the extent of the planar interface should be much larger than the transverse width $(a / \sin \xi)$ of the highly focused central pulse of the $\mathrm{X}$-wave. Since $a$ is inversely proportional to the maximum frequency of the spectrum and $\sin \xi$ is a small quantity, the latter condition can be rephrased as having a planar interface of extent much larger than all wavelengths contributing to the spectrum of the X-wave. Furthermore, one should note that in earlier investigations the case of infinite energy FWMs has been considered instead of X-waves. ${ }^{22,23}$ No attempt has been made at this stage to provide comparisons with recent work on the transmission and reflection of pulsed beams. $^{24,25}$ The latter are paraxial ultra-wide-band solutions to the scalar wave equation or Maxwell's equations, whereas $\mathrm{X}$-waves are exact ones. We would like to point out that our investigation is not exhaustive. Further extensions are needed in the case of multi-layered media, for investigations concerned with curved interfaces and when accounts must be taken of medium loss and dispersion mechanisms.

${ }^{1} \mathrm{P}$. Saari and K. Reivelt, "Evidence of X-shaped propagation-invariant localized light waves," Phys. Rev. Lett. 21, 4135-4138 (1997).

${ }^{2}$ J. Y. Lu and J. F. Greenleaf, "Experimental verification of nondiffracting X waves,' IEEE Trans. Ultrason. Ferroelectr. Freq. Control 39, 441-446 (1992).

${ }^{3}$ R. W. Ziolkowski, D. K. Lewis, and B. D. Cook, 'Experimental verification of the localized wave transmission effect,' Phys. Rev. Lett. 62, 147-150 (1989).

${ }^{4}$ R. W. Ziolkowski, "Localized wave physics and engineering,' Phys. Rev. A 44, 3960-3984 (1991).

${ }^{5}$ A. M. Shaarawi, I. M. Besieris, R. W. Ziolkowski, and S. M. Sedky, "Generation of approximate focus wave mode pulses from wide-band dynamic Gaussian aperture,'’ J. Opt. Soc. Am. A 12, 1954-1964 (1995).

${ }^{6}$ P. R. Stepanishen, “Acoustic bullets/transient Bessel beams: Near to far field transition via an impulse response approach,' J. Acoust. Soc. Am. 103, 1742-1751 (1998).

${ }^{7}$ A. A. Chatzipetros, A. M. Shaarawi, I. M. Besieris, and M. A. AbdelRahman, "Aperture synthesis of time-limited X-waves and analysis of their propagation characteristics,' J. Acoust. Soc. Am. 103, 2287-2295 (1998).

${ }^{8}$ D. Power, R. Donnelly, and R. MacIsaac, "Spherical scattering of superpositions of localized waves,'” Phys. Rev. E 48, 1410-1417 (1993).

${ }^{9}$ J. Y. Lu, M. Fatemi, and J. F. Greenleaf, "Pulsed-echo imaging with X wave,' Acoust. Imaging 22, 191-196 (1996).

${ }^{10}$ J. Y. Lu and J. F. Greenleaf, "Diffraction-limited beams and their applications for ultrasonic imaging and tissue characterization," Proc. SPIE 1733, 92-119 (1992).

${ }^{11}$ J. N. Brittingham, "Focus wave modes in homogeneous Maxwell equations: Transverse electric mode,'” J. Appl. Phys. 54, 1179-1189 (1983).

${ }^{12}$ R. W. Ziolkowski, "Exact solutions of the wave equation with complex source locations,'’ J. Math. Phys. 26, 861-863 (1985).

${ }^{13}$ A. Sezigner, "A general formulation of focus wave modes,' J. Appl. Phys. 57, 678 (1985). 
${ }^{14}$ I. M. Besieris, A. M. Shaarawi, and R. W. Ziolkowski, "A bidirectional traveling plane wave representation of exact solutions of the scalar wave equation,', J. Math. Phys. 30, 1254-1269 (1989).

${ }^{15}$ J. Y. Lu and J. F. Greenleaf, "Nondiffracting X waves-Exact solutions to free space scalar wave equation and their finite aperture realization,", IEEE Trans. Ultrason. Ferroelectr. Freq. Control 39, 19-31 (1992).

${ }^{16}$ J. Y. Lu and J. F. Greenleaf, "Sidelobe reduction for limited diffraction pulse-echo system,' IEEE Trans. Ultrason. Ferroelectr. Freq. Control 40, 735-746 (1993).

${ }^{17}$ J. Y. Lu and J. F. Greenleaf, "CComparison of sidelobes of limited diffraction beams and localized waves," Acoust. Imaging 22, 145-152 (1995).

${ }^{18}$ J. Y. Lu, "Producing bowtie limited diffraction beams with synthetic array experiment,' IEEE Trans. Ultrason. Ferroelectr. Freq. Control 42, 893-900 (1996).

${ }^{19}$ A. M. Shaarawi, S. M. Sedky, F. M. Taiel, R. W. Ziolkowski, and I. M. Besieris, "Spectral analysis of time-limited pulsed Gaussian wavefields,", J. Opt. Soc. Am. A 13, 1827-1836 (1996).

${ }^{20}$ A. M. Shaarawi, "Comparison of two localized wavefields generated from dynamic apertures,', J. Opt. Soc. Am. A 14, 1804-1816 (1997).

${ }^{21}$ I. Besieris, M. Abdel-Rahman, A. Shaarawi, and A. Chatzipetros, "Two fundamental representations of localized pulse solutions of the scalar wave equation,' Progress in Electromagnetics Research (PIER) 19, 1-48 (1998).

${ }^{22} \mathrm{P}$. Hillion, "How do focus wave modes propagate across a discontinuity in a medium?' Optik (Stuttgart) 93, 67-72 (1993).

${ }^{23}$ R. Donnelly and D. Power, "The behavior of electromagnetic localized waves at a planar interface,', IEEE Trans. Antennas Propag. 45, 580-591 (1997).
${ }^{24}$ E. Heyman and R. Ianconescu, "Pulsed beam reflection and transmission at a dielectric interface: two-dimensional fields,' IEEE Trans. Antennas Propag. AP-38, 1791-1800 (1990).

${ }^{25}$ E. Heyman, R. Strahilevitz, and D. Kosloff, "Pulsed beam reflection and transmission at a planar interface: Exact solutions and local models,', Wave Motion 18, 315-343 (1993).

${ }^{26}$ J. Fagerholm, A. T. Friberg, J. Huttunen, D. P. Morgan, and M. M. Salomaa, "Angular-spectrum representation of nondiffracting $\mathrm{X}$ waves,", Phys. Rev. E 54, 4347-4352 (1996).

${ }^{27}$ A. T. Friberg, J. Fagerholm, and M. M. Salomaa, "Space-frequency analysis of nondiffracting pulses,' Opt. Commun. 136, 207-212 (1997).

${ }^{28}$ I. S. Gradshteyn and I. M. Ryzhik, Tables of Integrals, Series and Products (Academic, New York, 1965).

${ }^{29}$ W. C. Elmore and M. A. Heald, Physics of Waves (Dover, New York, 1985).

${ }^{30}$ A. D. Pierce, Acoustics: An Introduction to its Physical Principles and Applications (McGraw-Hill, New York, 1981).

${ }^{31}$ A. M. Attiya, E. A. El-Diwany, A. Shaarawi, and I. Besieris, "Reflection and transmission of an electromagnetic X-wave in the presence of planarly layered media: The pulsed plane wave representation'" (unpublished).

${ }^{32}$ S. M. Sedky, A. M. Shaarawi, F. M. Taiel, and I. M. Besieris, "On the diffraction length of localized waves generated by dynamic apertures,' J. Opt. Soc. Am. A 13, 1719-1727 (1996).

${ }^{33}$ B. Hafizi and P. Sprangle, "Diffraction effects in directed radiation beams,'” J. Opt. Soc. Am. A 8, $705-717$ (1991). 